\title{
WORD MAPS ON PERFECT ALGEBRAIC GROUPS
}

\author{
NIKOLAI GORDEEV, BORIS KUNYAVSKIĬ, EUGENE PLOTKIN \\ To Boris Isaakovich Plotkin on the occasion of his 90th birthday
}

\begin{abstract}
We extend Borel's theorem on the dominance of word maps from semisimple algebraic groups to some perfect groups. In another direction, we generalize Borel's theorem to some words with constants. We also consider the surjectivity problem for particular words and groups, give a brief survey of recent results, present some generalizations and variations and discuss various approaches, with emphasis on new ideas, constructions and connections.
\end{abstract}

\section{INTRODUCTION}

The main object of the present paper is a word map $\widetilde{w}: G^{n} \rightarrow G$ defined for any word $w=w\left(x_{1}, \ldots, x_{n}\right)$ from the free group $F_{n}=\left\langle x_{1}, \ldots, x_{n}\right\rangle$ of rank $n$ and any group $G$. This map evaluates an $n$-tuple $\left(g_{1}, \ldots, g_{n}\right)$ by substituting in $w$ each $g_{i}$ instead of $x_{i}, g_{i}^{-1}$ instead of $x_{i}^{-1}$ and performing all group operations in $G$.

Our main interest is in studying the image of $\widetilde{w}$, in particular, the question when $\operatorname{Im} \widetilde{w}=G$, which, stated in simple-minded manner, consists in understanding whether the equation

$$
w\left(g_{1}, \ldots, g_{n}\right)=a
$$

with arbitrary right-hand side is solvable in $G$. (Note that the question on the structure of the fibres of word maps, which, in the same simple-minded manner, consists in studying the number of solutions of equation (0.1) for varying right-hand side, is not less interesting, leading to various results of equidistribution flavour, see, e.g., $\mathrm{LaS}, \mathrm{BK}$.)

In this paper we consider $G=\mathcal{G}(K)$ where $\mathcal{G}$ is a perfect algebraic group defined over a field $K$, which includes, as a particular case, semisimple groups. If $K$ is algebraically closed, we will identify $G$ with $\mathcal{G}$.

The starting point of all such considerations is Borel's theorem [Bo2] saying that for any non-identity word $w$ the induced word map $\widetilde{w}$ on any semisimple algebraic group is dominant. (For special words $w$ such as powers and commutators the question on the surjectivity of $\widetilde{w}$ was investigated much earlier.)

There are several eventual ways to strengthen Borel's theorem. First, one can try to extend the class of algebraic groups from semisimple groups to more general ones. In Section 2 we consider the case where $G$ is a perfect algebraic group. For certain subclasses of such groups we prove the dominance of arbitrary word maps.

Second, although in Borel's theorem $w$ is an arbitrary word, one can try to extend it to a more general case of words with constants. This line of research was started in our earlier papers [GKP1], GKP2]. In Section 5 we continue these considerations and prove some more dominance results.

Third, one can try to strengthen dominance results by proving the surjectivity of relevant word maps. Usually, to get statements of this kind, one has to pay a certain price by restricting attention either to particular words, or to particular groups, or to particular fields, or to any combination of above. In Section 3 we consider the first option and give a brief survey of results available for power words, commutators, and, more generally, Engel words. In Section 4 we consider the case of groups of Lie rank one. Here we

The research of the first author was financially supported by the Ministry of Education and Science of the Russian Federation, project 1.661.2016/1.4. The research of the second and third authors was supported by ISF grant $1623 / 16$ and the Emmy Noether Research Institute for Mathematics. The paper was finished when the second author visited the MPIM (Bonn). 
GORDEEV, KUNYAVSKIĬ, PLOTKIN

generalize some of recent results by Bandman and Zarhin [BZ and discuss various approaches which may eventually work in this case.

Throughout, if not stated otherwise, $K$ is an algebraically closed field. We postpone the investigation of word maps on algebraic groups over some special fields (particularly, local fields) to a forthcoming paper. We only note that here there is another type of problems, intermediate between the dominance and surjectivity: namely, one can ask about the structure of the image of the word map with respect to the natural topology arising from the ground field under consideration. This is important, for instance, for getting results of "Waring type" (we use the terminology introduced by Shalev, see, e.g., [Sh1]), when one is interested in representing every element of $G$ as a product of word values). Whereas in the case where $K$ is algebraically closed such results follow from the dominance in a straightforward way, much harder work is to be done over smaller fields. One can mention HLS for the case where $K$ is a local field as well as numerous papers devoted to finite groups of Lie type, see. e.g., [Sh1, [LST] and the references therein, as well as surveys $\mathrm{BGK}$, Sh2, Li]. Returning to the real case, note the striking difference arising for compact groups $G$, where the image of a word map may be arbitrarily small in the real topology Th (of course, remaining Zarsiki dense). Some more results for $p$-adic fields were obtained in [GS, AGKS, [HLS. See [Ku for a brief survey of results available over global fields.

\section{Notation and terminology}

$\mathbb{N}, \mathbb{Z}, \mathbb{R}, \mathbb{C}$ denote the set of natural numbers, the ring of integers, the fields of real and complex and numbers, respectively.

$\mathbb{A}_{K}^{n}$ is the affine space of dimension $n$ over the field $K$. We consider only affine varieties, which are closed irreducible subsets of $\mathbb{A}_{K}^{n}$. For a variety $X$ and a subset $Y \subset X$ by $\bar{Y}$ we denote the Zariski closure of $Y$ in $X$. In general, by topology we mean Zariski topology.

As mentioned above, the main object of our consideration is a perfect linear algebraic group $\mathcal{G}$ defined over a field $K$. This means that the commutator group $[\mathcal{G}, \mathcal{G}]$ coincides with $\mathcal{G}$. If $K$ is algebraically closed, we identify the group of $K$-points $G=\mathcal{G}(K)$ with $\mathcal{G}$.

The unipotent radical of $\mathcal{G}$ is denoted by $R_{u}(\mathcal{G})$.

If a group $H$ acts on a set $X$, the symbol $X^{H}$ stands for the set of $H$-invariant elements of $X$. $F_{n}=\left\langle x_{1}, \ldots, x_{n}\right\rangle$ is the free group of rank $n, F_{n}^{0}=F_{n}, F_{n}^{i}=\left[F_{n}^{i-1}, F_{n}^{i-1}\right]$.

When numbering our statements, we use capital letters for known results and numbers for new ones.

\section{Generalities on word maps on linear algebraic Groups}

1.1. $\operatorname{Aut}\left(\mathbf{F}_{\mathbf{n}}\right)$ - and $\operatorname{Aut}(\mathbf{G})$-invariance. Let $G$ be an abstract group.

Let us start with mentioning the following obvious (and well-known) fact:

$\operatorname{Im} \widetilde{w}$ is an $\operatorname{Aut}(G)$-invariant subset of $G$.

In another direction, let $\operatorname{Aut}\left(F_{n}\right)$ denote the group of automorphisms of $F_{n}$.

Proposition 1.1. If $w_{1}, w_{2} \in F_{n}$ lie in the same $\operatorname{Aut}\left(F_{n}\right)$-orbit, then for any group $G$ the maps $\widetilde{w}_{1}, \widetilde{w}_{2}: G^{n} \rightarrow G$ have the same image.

Proof. Indeed, any group homomorphism $\varphi: F_{n} \rightarrow G$ is determined by the $n$-tuple $\left(g_{1}=\varphi\left(x_{1}\right), \ldots, g_{n}=\right.$ $\left.\varphi\left(x_{n}\right)\right)$. Since for any $w \in F_{n}$ we have $\varphi(w)=\widetilde{w}\left(g_{1}, \ldots, g_{n}\right)$, the image of $\widetilde{w}$ coincides with the set $\{\varphi(w)\}_{\varphi \in \operatorname{Hom}\left(F_{n}, G\right)}$, whence the result.

Remark 1.2. Not too much is known about the invariance of $\operatorname{Im} \widetilde{w}$ with respect to other operations on $F_{n}$ and $G$. It would be interesting to divide words into equivalence classes with respect to certain invariance properties of $\operatorname{Im} \widetilde{w}$ for a given group $G$.

1.2. Semisimple algebraic groups. Consider the case where $G$ is a semisimple algebraic group.

The basic point for the investigation of word maps on semisimple algebraic groups is the following theorem of A. Borel [Bo2].

Theorem A. Let $G$ be a connected semisimple algebraic group, and let $1 \neq w \in F_{n}$. Then the map $\widetilde{w}: G^{n} \rightarrow G$ is dominant. 
Corollary 1.3. Let $w_{1} \in F_{n_{1}}, \ldots, w_{k} \in F_{n_{k}}, k>1$, be words without common letters, let $w=w_{1} w_{2} \ldots w_{k}$, and let $\widetilde{w}: G^{\sum_{i} n_{i}} \rightarrow G$ be the corresponding word map. Then $\operatorname{Im} \widetilde{w}=G$.

Proof. Since $w_{1}, \ldots, w_{k}$ are words without common letters, $\operatorname{Im} w=\operatorname{Im} w_{1} \operatorname{Im} w_{2} \cdots \operatorname{Im} w_{k}$. Theorem A implies that for every $i$ there is a non-empty open subset $U_{i}$ of $G$ which is contained in $\operatorname{Im} w_{i}$. Hence $U_{1} U_{2} \cdots U_{k} \subset \operatorname{Im} w$. But the product of any two non-empty open subsets of a linear algebraic group coincides with the whole group (see [Bo1, Ch. I, Prop. 1.3]).

Let $w \in F_{n}$, and let $\widetilde{w}: G^{n} \rightarrow G$ be the word map of semisimple algebraic groups. Then we may view the words $w_{1}=w\left(x_{1}^{(1)}, \ldots, x_{n}^{(1)}\right), \ldots, w_{k}=w\left(x_{1}^{(k)}, \ldots, x_{n}^{(k)}\right)$ as words in different variables. Applying Corollary [1.3, we get

Corollary 1.4. If $k>1$, then $(\operatorname{Im} \widetilde{w})^{k}=G$.

\section{WORD MAPS ON PERFECT ALGEBRAIC GROUPS}

It would be interesting to extend Theorem A to a wider class of algebraic groups. A natural step would be to assume $G=[G, G]$ to be a perfect group. However, this works only in particular cases which we are going to describe.

Throughout this section, $\mathcal{G}$ is a connected perfect linear algebraic group defined over an algebraically closed field $K$ of characteristic zero and $G=\mathcal{G}(K)$. (Recall that we identify $\mathcal{G}$ with $G$, see Introduction.)

2.1. General observations. Put $U=R_{u}(G)$. Then $G / U$ is a semisimple algebraic $K$-group [Bo1, 11.21] and by Mostow's Theorem [Mo] (see, e.g., [Ho, Th. VIII.4.3], [Co, Prop. 5.4.1] for modern exposition), there exists a closed linear algebraic subgroup $H$ of $G$ (called a Levi subgroup) isomorphic to $G / U$. (Equivalently, $G=H U$ is a semidirect product.) All Levi subgroups are conjugate. We fix one of them and denote by $H$ throughout below.

Let

$$
U_{1}=U, U_{2}=\left[U, U_{1}\right], \ldots, U_{i}=\left[U, U_{i-1}\right], \ldots, U_{r+1}=\{1\}
$$

be the lower central series of $U$, and let $V_{i}=U_{i} / U_{i+1}$ denote its quotients. Then we may view $V_{i}$ as a $K[H]$-module (indeed, the action of $H$ on $V_{i}$ induced by conjugation of $U$ by elements of $G$ is $K$-linear because char $K=0)$.

Definition 2.1. We say that a $K[H]$-module $M$ is augmentative if it has no $K[H]$-quotients $M / M^{\prime}$ on which $H$ acts trivially.

If $G$ is a perfect group, $V_{1}$ is an augmentative $K[H]$-module GS, Gor3.

Definition 2.2. We say that $G$ is a firm perfect group if $V_{i}$ is an augmentative $K[H]$-module for every $i$.

Remark 2.3. Note that if the nilpotency class of $U$ is equal to one, that is, if $U$ is an abelian group, then any perfect group $G$ is firm.

Definition 2.4. We say that $G$ is a strictly firm perfect group if $V_{i}^{T}=\{0\}$ for every $i$ where $T$ is a maximal torus of $G$.

Example 2.5. Let $G$ be a simple classical algebraic group (that is of type $A_{r}, B_{r}, C_{r}$, or $D_{r}$ ), and let $\Pi=\left\{\alpha_{1}, \ldots, \alpha_{r}\right\}$ be its standard simple root system (with the notation of [Bou, Planches]). Further, let $P_{k}=P_{X}$ be the standard parabolic subgroup of $G$ which corresponds to the set of simple roots $X=\Pi \backslash\left\{\alpha_{k}\right\}$. For the case $A_{r}, r>1$, the group $\left[P_{k}, P_{k}\right]$ is a strictly firm perfect group for every $k$. For all remaining cases, the same is true for $r>2$ and $k>2$. Indeed, it is enough to show that there are no positive roots $\beta$ orthogonal to every root $\alpha_{i} \in X$. This follows, in its turn, from the following observation: since $k>2$, such a root $\beta$ is orthogonal to $\alpha_{1}=\epsilon_{1}-\epsilon_{2}$ and $\alpha_{2}=\epsilon_{2}-\epsilon_{3}$, and therefore $\beta$ is either $\epsilon_{i} \pm \epsilon_{j}$, or $\epsilon_{i}$, or $2 \epsilon_{i}$, where $3<i \leq k, j>i$. But then $\beta$ is not orthogonal to $\alpha_{i-1}=\epsilon_{i-1}-\epsilon_{i}$.

Theorem 2.6. Let $G$ be a connected perfect algebraic group defined over an algebraically closed field $K$ of characteristic zero. Then 
(i) if $G$ is strictly firm, then for any $1 \neq w \in F_{n}$ the map $\widetilde{w}: G^{n} \rightarrow G$ is dominant;

(ii) if $G$ is firm, then for any $w=w_{1}\left(x_{1}, \ldots, x_{n}\right) w_{2}\left(y_{1}, \ldots, y_{k}\right) \in F_{n+k}, w_{1}, w_{2} \neq 1$, the word map $\widetilde{w}: G^{n+k} \rightarrow G$ is dominant.

Proof.

(i) Let $\mathfrak{d}: U^{m} \rightarrow U$ be a map such that for every $i$

(I) $\mathfrak{d}\left(U_{i}^{m}\right) \subset U_{i}$;

(II) for any two $m$-tuples $\left(u_{1}, u_{2}, \ldots, u_{m}\right) \in U_{i}^{m}$ and $\left(u_{1}^{\prime}, u_{2}^{\prime}, \ldots, u_{m}^{\prime}\right) \in U_{j}^{m}, j>i$ we have

$$
\mathfrak{d}\left(u_{1} u_{1}^{\prime}, u_{2} u_{2}^{\prime}, \ldots, u_{m} u_{m}^{\prime}\right) \equiv \mathfrak{d}\left(u_{1}, u_{2}, \ldots, u_{m}\right) \mathfrak{d}\left(u_{1}^{\prime}, u_{2}^{\prime}, \ldots, u_{m}^{\prime}\right) \quad\left(\bmod U_{j+1}\right) .
$$

Then we may consider the induced maps $\mathfrak{d}_{i}: V_{i}^{m} \rightarrow V_{i}$ given by

$$
\mathfrak{d}_{i}\left(v_{1}, \ldots, v_{m}\right) \equiv \mathfrak{d}\left(u_{1}, u_{2}, \ldots, u_{m}\right) \quad\left(\bmod U_{i+1}\right)
$$

where $\left(u_{1}, u_{2}, \ldots, u_{m}\right)$ is an $m$-tuple of preimages of $\left(v_{1}, \ldots, v_{m}\right) \in V_{i}^{m}=\left(U_{i} / U_{i+1}\right)^{m}$ in $U_{i}^{m}$.

Lemma 2.7. Let $\mathfrak{d}: U^{m} \rightarrow U$ be a map satisfying conditions (I), (II). If $\mathfrak{d}_{i}\left(V_{i}^{m}\right)=V_{i}$ for every $i$, then $\mathfrak{d}\left(U^{m}\right)=U$.

Proof. Let $u \in U_{i} \backslash U_{i+1}$. Since $\mathfrak{d}_{i}\left(V_{i}^{m}\right)=V_{i}$, we have $\left(u_{1}, u_{2}, \ldots, u_{m}\right) \in U_{i}^{m}$ such that $\mathfrak{d}\left(u_{1}, u_{2}, \ldots, u_{m}\right)=$ $u u^{\prime}$ where $u^{\prime} \in U_{i+1}$. Let $u^{\prime} \in U_{j} \backslash U_{j+1}$ for some $j>i$. Then there is $\left(u_{1}^{\prime}, u_{2}^{\prime}, \ldots, u_{m}^{\prime}\right) \in U_{j}^{m}$ such that $\mathfrak{d}\left(u_{1}^{\prime}, u_{2}^{\prime}, \ldots, u_{m}^{\prime}\right) \equiv u^{\prime-1}\left(\bmod U_{j+1}\right)$. Condition (II) implies that $\mathfrak{d}\left(u_{1} u_{1}^{\prime}, u_{2} u_{2}^{\prime}, \ldots, u_{m} u_{m}^{\prime}\right)=u u^{\prime \prime}$ where $u^{\prime \prime} \in U_{j+1}$. Acting this way, we can find a preimage of $u$ in $U_{i}^{m}$.

Since $G$ is a strictly firm perfect group, there exists a non-empty open subset $X \subset T$ such that $\left[t^{-1}, V_{i}\right]=V_{i}$ for every $t \in X$ and every $i$ because $V_{i}$ is a finite-dimensional $K[H]$-module.

Let $\widetilde{w}_{H}: H^{n} \rightarrow H$ be the map corresponding to the same word $w$. Then $\widetilde{w}_{H}$ is dominant according to the Borel Theorem, and therefore there exists an open subset $Y \subset T$ such that every element $t \in Y$ has a non-empty preimage $\widetilde{w}_{H}^{-1}(t)$.

Let $t \in X \cap Y$. Then

(a) there is an $n$-tuple $\left(h_{1}, \ldots, h_{n}\right) \in H^{n}$ such that $\widetilde{w}_{H}\left(h_{1}, \ldots, h_{n}\right)=t$;

(b) $\left[t^{-1}, V_{i}\right]=V_{i}$ for every $i$.

We have $t \in \operatorname{Im} \widetilde{w}$ because $G$ contains $H$. Then for every $u \in U$ we have $u t u^{-1} \in \operatorname{Im} \widetilde{w}$. Thus, $t\left[t^{-1}, u\right] \in \operatorname{Im} \widetilde{w}$ for every $u$. Since $\left[t^{-1}, V_{i}\right]=V_{i}$ for every $i$, condition (b) and Lemma 2.7 (for $m=1$ and $\left.\mathfrak{d}(u)=\left[t^{-1}, u\right]\right)$ imply that for every $u^{\prime} \in U$ there exists $u \in U$ such that $\left[t^{-1}, u\right]=u^{\prime}$. Hence $t U \in \operatorname{Im} \widetilde{w}$ for every $t$ belonging to a non-empty open subset $X \cap Y$. Then $\operatorname{Im} \widetilde{w}$ is dense in $G$.

(ii) We need the following

Lemma 2.8. If statement (ii) holds for $K=\mathbb{C}$, then it holds for every algebraically closed field $K$ of characteristic zero.

Proof. Since char $K=0$, there exists an extension $F / K$ such that $\mathbb{C} \subset F$. Note that $G=\mathcal{G}(K)$ and $\mathcal{G}(\mathbb{C})$ are dense subgroups of $\mathcal{G}(F)\left[\right.$ Bo1, 18.3]. Hence if we prove that the image of $\widetilde{w}: \mathcal{G}(\mathbb{C})^{n+k} \rightarrow \mathcal{G}(\mathbb{C})$ is dense, we also get the density of the image of the map $\widetilde{w}: \mathcal{G}(K)^{n+k} \rightarrow \mathcal{G}(K)$.

Now we assume $G=\mathcal{G}(\mathbb{C})$. We denote by $H_{c}$ a maximal compact subgroup of $H$. It is a real compact Lie group. Consider the action of $H_{c}$ on $V_{i}$ for a fixed $i$. Since $G$ is a firm group, the natural representation $\mathfrak{i}: H_{c} \rightarrow \mathrm{GL}\left(V_{i}\right)$ is not trivial, and therefore the image $\mathfrak{i}\left(H_{c}\right)$ is a non-trivial compact subgroup of $\mathrm{SL}\left(V_{i}\right)$ (recall that $\mathcal{G}$ is a connected perfect group). To ease the notation, we will identify the group $\mathfrak{i}\left(H_{c}\right) \leq \mathrm{SL}\left(V_{i}\right)$ with $H_{c}$. We may consider a positive-definite hermitian form $\varphi$ on $V_{i}$ and the group $\mathrm{SU}\left(V_{i}\right)$, which is a maximal compact subgroup of $\mathrm{SL}\left(V_{i}\right)$. We may and will assume $H_{c} \leq \mathrm{SU}\left(V_{i}\right)$.

Recall that by Mostow's Theorem, $G$ is a semidirect product $H U$ where $H \leq G$ is a (fixed) Levi subgroup of $G$. The words $w_{1}, w_{2}$ induce dominant maps $\widetilde{w}_{1}: H^{n} \rightarrow H, \widetilde{w}_{2}: H^{k} \rightarrow H$. By the same arguments as above, we can now get a non-empty open set $X \cap Y \subset T$ such that for every $t \in X \cap Y$ the following properties hold: 
(a) there is an $n$-tuple $\left(h_{1}, \ldots, h_{n}\right) \in H^{n}$ (respectively, $\left.\left(h_{1}, \ldots, h_{k}\right) \in H^{k}\right)$ such that $\widetilde{w}_{1}\left(h_{1}, \ldots, h_{n}\right)=t\left(\right.$ respectively, $\left.\widetilde{w}_{2}\left(h_{1}, \ldots, h_{k}\right)=t\right) ;$

(c) $\left[t^{-1}, V_{i}\right]=\left[T, V_{i}\right]$ for every $i$.

Further, there exists a maximal torus $T^{\prime} \leq H$ such that $\overline{\left\langle T, T^{\prime}\right\rangle}=H$.

Indeed, there are only finitely many connected proper closed subgroups $\left\{\Gamma_{i}\right\}$ of $H$ which contain a given maximal torus $T$ [Bo1, 9.4]. Since the set of all semisimple elements is dense in $G$, there is a maximal torus $T^{\prime} \leq H$ such that $T^{\prime} \nsubseteq \Gamma_{i}$ for every $i$. Let $\Delta=\overline{\left\langle T, T^{\prime}\right\rangle}$, then the identity component $\Delta^{0}$ contains $T^{\prime}$. Then we have $\Delta^{0} \neq \Gamma_{i}$ for every $i$ but $T \leq \Delta^{0}$. This implies $\Delta=\Delta^{0}=H$.

So for every $t^{\prime} \in T^{\prime}$ we have $\overline{\left\langle t^{\prime-1} T t^{\prime}, T^{\prime}\right\rangle}=H$. Hence there is an open set $Z \subset H \times H$ such that if $\left(t, t^{\prime}\right) \in Z$, then

(1) $t \in T, t^{\prime} \in T^{\prime}$, where $T, T^{\prime}$ is a pair of maximal tori of $H$ such that $\overline{\left\langle T, T^{\prime}\right\rangle}=H$;

(2) there exist $\left(h_{1}, \ldots, h_{n}\right) \in H^{n},\left(h_{1}^{\prime}, \ldots, h_{k}^{\prime}\right) \in H^{k}$ such that

$$
\widetilde{w}_{1}\left(h_{1}, \ldots, h_{n}\right)=t, \widetilde{w}_{2}\left(h_{1}^{\prime}, \ldots, h_{k}^{\prime}\right)=t^{\prime} ;
$$

(3) $\left[t^{-1}, V_{i}\right]=\left[T, V_{i}\right],\left[t^{\prime-1}, V_{i}\right]=\left[T^{\prime}, V_{i}\right]$ for every $i$.

Indeed, obviously, a general pair in $H \times H$ satisfies condition (1). Conditions (2) and (3) follow from (a) and (c).

Since $H_{c}$ is Zariski dense in $H$, we may assume that maximal compact tori $T_{c} \leq T, T_{c}^{\prime} \leq T^{\prime}$ are contained in $H_{c}$ and there is a Zariski dense subset $Z_{c} \subset Z, Z_{c} \subset H_{c} \times H_{c}$ such that for every $\left(t, t^{\prime}\right) \in Z_{c}$ conditions (1), (2), (3) are satisfied with $T$ replaced by $T_{c}$ and $T^{\prime}$ replaced by $T_{c}^{\prime}$.

Let now $u, u^{\prime} \in U_{i}$. Then

$$
\begin{gathered}
\widetilde{w}\left(u h_{1} u^{-1}, \ldots, u h_{n} u^{-1} ; v^{\prime} h_{1}^{\prime} u^{\prime-1}, \ldots, u^{\prime} h_{k}^{\prime} u^{\prime-1}\right)=u t u^{-1} u^{\prime} t^{\prime} u^{\prime-1}=t\left[t^{-1}, u\right] t^{\prime}\left[t^{\prime-1}, u^{\prime}\right]= \\
t t^{\prime}\left(t^{\prime-1}\left[t^{-1}, u\right] t^{\prime}\right)\left[t^{\prime-1}, u^{\prime}\right]=t t^{\prime}\left(\left[t^{\prime-1} t^{-1} t^{\prime}, t^{\prime-1} u t^{\prime}\right]\right)\left(\left[t^{\prime-1}, u^{\prime}\right]\right) \in \operatorname{Im} \widetilde{w} .
\end{gathered}
$$

Consider the map

$$
\mathfrak{d}: U \times U \rightarrow U
$$

given by $\mathfrak{d}\left(u, u^{\prime}\right)=\left[t^{\prime-1} t^{-1} t^{\prime}, t^{\prime-1} u t^{\prime}\right]\left[t^{\prime-1}, u^{\prime}\right]$. Obviously, the map $\mathfrak{d}$ satisfies condition (I). Let us check (II). We have the identity $[a, b c]=[a, b] b[a, c] b^{-1}$ that becomes $[a, b c]=[a, b][a, c]$ in the case when $b$ commutes with $[a, c]$. Further, let $u, u^{\prime} \in U_{i}, v, v^{\prime} \in U_{j}$ where $j>i$, and let $\bar{u}, \bar{u}^{\prime}, \bar{v}, \bar{v}^{\prime}$ be the images of $u, u^{\prime} v, v^{\prime}$ in $U / U_{j+1}$. Note that $\bar{v}, \bar{v}^{\prime}$, as well as elements of the form $s \bar{v} s^{-1}, s \bar{v}^{\prime} s^{-1},[s, \bar{v}],\left[s, \bar{v}^{\prime}\right]$, belong to the centre of $U / U_{j+1}$. Then using the commutator identity $[a, b c]=[a, b][a, c]$, we get property (II) for $\mathfrak{d}$. Then $\mathfrak{d}$ induces bilinear maps $\mathfrak{d}_{i}: V_{i} \times V_{i} \rightarrow V_{i}$ given by

$$
\mathfrak{d}_{i}\left(v, v^{\prime}\right)=\left(t^{\prime-1} t^{-1} t^{\prime}(v)-v\right)+\left(t^{\prime-1}\left(v^{\prime}\right)-v^{\prime}\right)
$$

where $v, v^{\prime} \in V_{i}$ are the images of $t^{\prime-1} u t^{\prime}, u^{\prime} \in U_{i}$ (here we change the multiplicative notation for the operation in $U_{i}$ to the additive notation for the operation in $\left.V_{i}=U_{i} / U_{i+1}\right)$.

Since $\overline{\left\langle T_{c}, T_{c}^{\prime}\right\rangle}=H$, we have $\overline{\left\langle t^{\prime-1} T_{c} t^{\prime}, T_{c}^{\prime}\right\rangle}=H$. Therefore, if $t^{\prime-1} t^{-1} t^{\prime}(l)-l=0$ and $t^{\prime-1}(l)-l=0$, then condition (3) implies that $l \in V_{i}^{H}$. Since char $K=0$ and $G$ is a firm perfect group, $V_{i}^{H}=\{0\}$. Thus, we get

$$
\left\{\begin{array}{l}
t^{\prime-1} t^{-1} t^{\prime}(l)-l=0 \\
\text { and } \\
t^{\prime-1}(l)-l=0
\end{array} \quad \Rightarrow l=0 .\right.
$$

From (3) we have

$$
\operatorname{Im} \mathfrak{d}_{i}=\left[t^{\prime-1} T t^{\prime}, V_{i}\right]+\left[T^{\prime}, V_{i}\right]
$$

On the other hand,

$$
V_{i}^{t^{\prime-1}} T_{c} t^{\prime}=V_{i}^{t^{\prime-1}} T t^{\prime}, V_{i}^{T_{c}^{\prime}}=V_{i}^{T^{\prime}}\left[t^{\prime-1} T t^{\prime}, V_{i}\right]=\left[t^{\prime-1} T_{c} t^{\prime}, V_{i}\right],\left[T^{\prime}, V_{i}\right]=\left[T_{c}^{\prime}, V_{i}\right] .
$$

If $l, l^{\prime}$ are vectors of the hermitian space $V_{i}$ such that $t(l)=\alpha l, \alpha \in \mathbb{C}, \alpha \neq 1$ and $t\left(l^{\prime}\right)=l^{\prime}$ for some $t \in T_{c}$, then $\varphi\left(l, l^{\prime}\right)=0$ (the same is true for the eigenvectors of the unitary transformation $t^{\prime}$ ). The vector space $V_{i}^{t^{\prime-1}} T_{c} t^{\prime}$ consists of the zero weight vectors of $t^{\prime-1} T_{c} t^{\prime}$, the vector space $\left[t^{\prime-1} T_{c} t^{\prime}, V_{i}\right]$ is spanned by 
all non-zero weight vectors of $t^{\prime-1} T_{c} t^{\prime}$, and the vector space $V_{i}^{t^{\prime-1}} T_{c} t^{\prime}$ is orthogonal to the vector space $\left[t^{\prime-1} T_{c} t^{\prime}, V_{i}\right]$. The same holds for the vector spaces $V_{i}^{T_{c}^{\prime}}=\left[T_{c}^{\prime}, V_{i}\right]$. Hence

$$
V_{i}^{t^{\prime-1}} T_{c} t^{\prime}=\left[t^{\prime-1} T_{c} t^{\prime}, V_{i}\right]^{\perp}, \quad V_{i}^{T_{c}^{\prime}}=\left[T_{c}^{\prime}, V_{i}\right]^{\perp}
$$

where $X^{\perp}$ is the orthogonal complement to $X$.

Suppose that

$$
L_{i}=\left[t^{\prime-1} T t^{\prime}, V_{i}\right]+\left[T^{\prime}, V_{i}\right] \neq V_{i} .
$$

Since $L_{i}$ is a subspace of the hermitian space $V_{i}$ with a positive-definite hermitian form $\varphi$, we have

$$
V_{i}=L_{i}+L_{i}^{\perp}
$$

From (2.6) and (2.7) we conclude that $L_{i}^{\perp} \neq 0$. But

$$
\begin{aligned}
& L_{i}^{\perp} \stackrel{\text { (2.6) }}{\leq}\left[t^{\prime-1} T t^{\prime}, V_{i}\right]^{\perp} \cap\left[T^{\prime}, V_{i}\right]^{\perp} \stackrel{(2.4)}{=}\left[t^{\prime-1} T_{c} t^{\prime}, V_{i}\right]^{\perp} \cap\left[T_{c}, V_{i}\right]^{\perp}=
\end{aligned}
$$

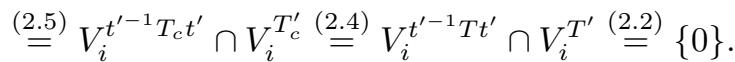

This contradicts assumption (2.6). Hence $L_{i}=\left[t^{\prime-1} T t^{\prime}, V_{i}\right]+\left[T^{\prime}, V_{i}\right]=V_{i}$, and from (2.3) we get

$$
\operatorname{Im} \mathfrak{d}_{i}=L_{i}=\left[t^{\prime-1} T t^{\prime}, V_{i}\right]+\left[T^{\prime}, V_{i}\right]=V_{i} .
$$

Note that for chosen $t, t^{\prime} \in H_{c}$ we have equality (2.8) for every $i$. Hence Lemma 2.7 (with $m=2$ ) implies that the map $\mathfrak{d}: U \times U \rightarrow U$ given by $\mathfrak{d}\left(u, u^{\prime}\right)=\left[t^{\prime-1} t^{-1} t^{\prime}, t^{\prime-1} u t^{\prime}\right]\left[t^{\prime-1}, u^{\prime}\right]$ is surjective. Now condition (2) for the choice of $t, t^{\prime}$ and (2.1) imply that there is a dense subset $I \subset H_{c}$ such that $I=\left\{t t^{\prime} \quad \mid \quad\left(t, t^{\prime}\right) \in Z_{c}\right\}$ and for every $s \in I$ we have $s U \subset \operatorname{Im} \widetilde{w}$ (this follows from (2.1) and (2.8)). Hence $I U \subset \operatorname{Im} \widetilde{w}$ and $\overline{I U}=G$. Thus, $\widetilde{w}$ is a dominant map.

Corollary 2.9. Let $G$ be a perfect algebraic group over an algebraically closed field $K$ of characteristic zero.

(i) If $G$ is strictly firm, then for any $1 \neq w \in F_{n}$ we have $(\operatorname{Im} \widetilde{w})^{2}=\mathcal{G}$.

(ii) If $G$ is firm, then for any $1 \neq w \in F_{n}$ we have $(\operatorname{Im} \widetilde{w})^{4}=\mathcal{G}$.

Remark 2.10. It would be interesting to obtain analogues of (at least some of the) results of this section for Lie polynomials on perfect Lie algebras, in the spirit of [BGKP].

2.2. Special cases. In this section we consider more carefully some special word maps $\widetilde{w}: G^{n} \rightarrow$ $G, \widetilde{w}_{H}: H^{n} \rightarrow H$ on a connected perfect algebraic group $G$ and its quotient $H=G / U$. Throughout this section we assume $\operatorname{char} K=0$.

Below we consider the linear action of $G$ (induced by conjugation) on vector spaces $V_{i}=U_{i} / U_{i+1}$.

Let $\mathfrak{h}=\left(h_{1}, \ldots, h_{n}\right) \in H^{n}$ be an $n$-tuple such that $\widetilde{w}_{H}\left(h_{1}, \ldots, h_{n}\right)=s \in H$. Let $\left(u_{1}, \ldots, u_{n}\right) \in U^{n}$. Then there is a map $\mathfrak{d}_{\mathfrak{h}}: U^{n} \rightarrow U$ such that

$$
\widetilde{w}\left(h_{1} u_{1}, h_{2} u_{2}, \ldots, h_{n} u_{n}\right)=\underbrace{\widetilde{w}\left(h_{1}, \ldots, h_{n}\right)}_{=s \in H} \underbrace{\mathfrak{d}_{\mathfrak{h}}\left(u_{1}, \ldots, u_{n}\right)}_{\in U}
$$

(the functions $\mathfrak{d}_{\mathfrak{h}}\left(z_{1}, \ldots, z_{n}\right)$ can be expressed by formulas containing the variables $z_{i}$ and the operators of conjugation by the elements $h_{i}^{ \pm 1}$ ). Thus, for a fixed $s \in H$ the set of all elements in $\operatorname{Im} \widetilde{w}$ whose projection onto $H$ is equal to $s$ is the set

$$
\left\{s \mathfrak{d}_{\mathfrak{h}}\left(u_{1}, \ldots, u_{n}\right) \mid\left(u_{1}, \ldots, u_{n}\right) \in U^{n}, \mathfrak{h} \in \widetilde{w}_{H}^{-1}(s)\right\} .
$$

Then

$$
\widetilde{w} \text { is a dominant map } \Leftrightarrow \overline{\bigcup_{\mathfrak{h} \in \widetilde{w}_{H}^{-1}(s)} \operatorname{Im} \mathfrak{d}_{\mathfrak{h}}}=U \text { for every } s \text { from some open subset of } H \text {, }
$$

$$
\widetilde{w} \text { is a surjective map } \Leftrightarrow \bigcup_{\mathfrak{h} \in \widetilde{w}_{H}^{-1}(s)} \operatorname{Im} \mathfrak{d}_{\mathfrak{h}}=U \text { for every } s \in H \text {. }
$$


Example 2.11. Let $w=x^{m}, h \in H$. Then

$$
w(h u)=(h u)^{m}=h^{m}\left(h^{-m+1} u h^{m-1}\right)\left(h^{-m+2} u h^{m-2}\right) \cdots\left(h^{-m+(m-1)} u h^{m-(m-1)}\right) u
$$

and therefore

$$
\mathfrak{d}_{\mathfrak{h}}(u)=\left(h^{-m+1} u h^{m-1}\right)\left(h^{-m+2} u h^{m-2}\right) \cdots\left(h^{-m+(m-1)} u h^{m-(m-1)}\right) u .
$$

Example 2.12. Let $w=[x, y], \mathfrak{h}=\left(h_{1}, h_{2}\right) \in H^{2}$. Then we have

$$
\begin{gathered}
{\left[h_{1} u_{1}, h_{2} u_{2}\right]=h_{1} u_{1} h_{2} u_{2} u_{1}^{-1} h_{1}^{-1} u_{2}^{-1} h_{2}^{-1}=} \\
=h_{1} h_{2} h_{1}^{-1} h_{2}^{-1}\left(h_{2} h_{1} h_{2}^{-1} u_{1} h_{2} h_{1}^{-1} h_{2}^{-1}\right)\left(h_{2} h_{1} u_{2} u_{1}^{-1} h_{1}^{-1} h_{2}^{-1}\right)\left(h_{2} u_{2}^{-1} h_{2}^{-1}\right)
\end{gathered}
$$

and therefore

$$
\mathfrak{d}_{\mathfrak{h}}\left(u_{1}, u_{2}\right)=\left(h_{2} h_{1} h_{2}^{-1} u_{1} h_{2} h_{1}^{-1} h_{2}^{-1}\right)\left(h_{2} h_{1} u_{2} u_{1}^{-1} h_{1}^{-1} h_{2}^{-1}\right)\left(h_{2} u_{2}^{-1} h_{2}^{-1}\right) .
$$

Theorem 2.13. Let $G$ be a firm perfect algebraic group. If $w \notin\left[F_{n}, F_{n}\right]$ and char $K=0$, then the map $\widetilde{w}: G^{n} \rightarrow G$ is dominant.

Proof. The restriction of $\mathfrak{d}_{\mathfrak{h}}$ to $U_{i}$ induces the maps

$$
\mathfrak{d}_{\mathfrak{h}}(i): V_{i}=U_{i} / U_{i+1} \rightarrow V_{i}=U_{i} / U_{i+1} .
$$

Lemma 2.14. For every $i$ the map $\mathfrak{d}_{\mathfrak{h}}(i): V_{i}=U_{i} / U_{i+1} \rightarrow V_{i}$ is $K$-linear and can be written as a sum of $K$-linear maps

$$
\mathfrak{d}_{\mathfrak{h}}(i)=\partial_{\mathfrak{h}}(i 1)+\partial_{\mathfrak{h}}(i 2)+\cdots+\partial_{\mathfrak{h}}(i n)
$$

where $\partial_{\mathfrak{h}}(i j)\left(v_{1}, \ldots, v_{n}\right)=\mathfrak{d}_{h}(i)\left(0, \ldots, 0, v_{j}, 0, \ldots, 0\right)$.

Proof. Let $\left(v_{1}, \ldots, v_{n}\right) \in V_{i}^{n}$, and let $u_{i}$ be a preimage of $v_{i}$ in $U_{i}$. We have

$$
\mathfrak{d}_{\mathfrak{h}}(i)\left(v_{1}, \ldots, v_{n}\right) \stackrel{\text { def }}{=} \mathfrak{d}_{\mathfrak{h}}\left(u_{1}, \ldots, u_{n}\right) \quad\left(\bmod U_{i+1}\right) .
$$

On the other hand, $\mathfrak{d}_{\mathfrak{h}}\left(u_{1}, \ldots, u_{n}\right)$ is a product of elements of the form

$$
h_{l_{1}}^{ \pm 1} h_{l_{2}}^{ \pm 1} \cdots h_{l_{k}}^{ \pm 1} u_{j} h_{l_{k}}^{\mp 1} \ldots h_{l_{2}}^{\mp 1} h_{l_{1}}^{\mp 1} .
$$

Conjugation of elements of $U_{i}$ by $h(h \in H)$ induces a $K$-linear map $h: V_{i} \rightarrow V_{i}$. Thus, we have

$$
\mathfrak{d}_{\mathfrak{h}}(i)\left(v_{1}, \ldots, v_{n}\right)=\sum_{j=1}^{n} \underbrace{\left(\text { a sum of elements of the form } h_{l_{1}}^{ \pm 1} h_{l_{2}}^{ \pm 1} \cdots h_{l_{k}}^{ \pm 1}\left(v_{j}\right)\right)}_{:=\partial_{\mathfrak{h}}(i j)\left(v_{1}, \ldots, v_{n}\right)} .
$$

Let now $w \notin\left[F_{n}, F_{n}\right]$. Then there is a variable $x_{i}$ such that $w\left(1, \ldots, 1, x_{i}, 1, \ldots, 1\right)=x_{i}^{m}, m \neq 0$. Hence it is enough to prove the statement for the case $w=x^{m}$. Example 2.11] and Lemma 2.14 show that for every $h \in H, \mathfrak{h}=(h)$ and every $i$ we have

$$
\mathfrak{d}_{\mathfrak{h}}(i)(v)=h^{m-1}(v)+h^{m-2}(v)+\cdots+h(v)+v=\left(h^{m-1}+h^{m-2}+\cdots+h+1\right)(v) .
$$

The linear operator $\left(h^{m-1}+h^{m-2}+\cdots+h+1\right)$ on $V_{i}$ is not invertible only in the cases when the operator $h$ has eigenvalues $\alpha=\sqrt[m]{1} \neq 1$. Hence there is an open subset $X \subset H$ such that for every $h \in X$ and every $i$ the linear operator $\left(h^{m-1}+h^{m-2}+\cdots+h+1\right)$ is invertible. Thus, for $h \in X$ we have $\mathfrak{d}_{\mathfrak{h}}(i)\left(V_{i}\right)=V_{i}$ for every $i$. Lemma 2.7 implies $\mathfrak{d}_{\mathfrak{h}}(U)=U$. Hence $s U \in \operatorname{Im} \widetilde{w}$ for every $s \in X$, and therefore $X U \subset \operatorname{Im} \widetilde{w}$. Thus, $\widetilde{w}$ is dominant.

Theorem 2.15. Let $G$ be a firm perfect algebraic group, and let $w=[x, y]$. Then the word map $\widetilde{w}: G^{2} \rightarrow$ $G$ is dominant. 
GORDEEV, KUNYAVSKIĬ, PLOTKIN

Proof. Example 2.12 shows that for every $i$, for every $\mathfrak{h}=\left(h_{1}, h_{2}\right) \in H \times H$ and $\left(v_{1}, v_{2}\right) \in V_{i}=U_{i} / U_{i+1}$, we have

$$
\begin{gathered}
\mathfrak{d}_{\mathfrak{h}}(i)\left(v_{1}, v_{2}\right)=h_{2} h_{1} h_{2}^{-1}\left(v_{1}\right)+h_{2} h_{1}\left(v_{2}\right)-h_{2} h_{1}\left(v_{1}\right)-h_{2}\left(v_{2}\right)= \\
=h_{2}\left[\left(h_{1} h_{2}^{-1} h_{1}^{-1}\left(h_{1}\left(v_{1}\right)\right)-h_{1}\left(v_{1}\right)\right)+\left(h_{1}\left(v_{2}\right)-v_{2}\right)\right] .
\end{gathered}
$$

The same arguments as in the proof of Theorem 2.6 show that for every $i$ we have $\mathfrak{d}_{\mathfrak{h}}(i)\left(V_{i}^{2}\right)=V_{i}$ for any pair $\left(h_{1}, h_{2}\right)$ belonging to a dense subset $X \subset H^{2}$, and therefore $\mathfrak{d}_{\mathfrak{h}}\left(U^{2}\right)=U$. Then the set $Y=\left\{\left[h_{1}, h_{2}\right] \mid h_{1}, h_{2} \in X\right\}$ is dense in $H$ and $s U \in \operatorname{Im} \widetilde{w}$ for every $s \in Y$. This proves the statement.

Remark 2.16. It would be interesting to investigate the extendability of the results of this section to the case where char $K=p>0$. There are (at least) two subtleties, even when $K$ is algebraically closed: first, the representation $G=H U$ as a semidirect product may not exist; second, the action on $V=G / U$ may not be linear. See $\mathrm{McN1}$, $\mathrm{McN2}$ for details.

To finish with general considerations, let us mention the intriguing question on the existence of eventual obstructions to the extendability of Borel's dominance theorem to perfect groups.

Question 2.17. Do there exist a field $K$, a connected perfect $K$-group $\mathcal{G}$ and a non-identity word $w \in F_{n}$ such that the word map $\widetilde{w}:(\mathcal{G}(K))^{n} \rightarrow \mathcal{G}(K)$ is not dominant?

\section{SurJeCtivity OF SPECIAL WORD MAPS}

For certain words we know more than in general case, particularly, regarding the surjectivity of the corresponding word map. In this section we give a brief overview of the power words and Engel words.

3.1. The power map $w=x^{m}$. In the case of semisimple algebraic groups we have a complete answer.

Theorem B. (Steinberg Stei], Chatterjee [Ch2]-Ch3]) Let $K$ be an algebraically closed field of characteristic exponent $p$ (i.e., $p=1$ if char $K=0$ and $p=\operatorname{char} K$ otherwise). Let $G$ be a connected semisimple algebraic group. Then the map $x \mapsto x^{m}$ is surjective on $G$ if and only if $m$ is prime to prz, where $z$ is the order of the centre of $G$ and $r$ is the product of "bad" primes.

In particular, one can guarantee that $m$-th roots can be extracted in an arbitrary connected semisimple group of adjoint type over $\mathbb{C}$ if and only if $m$ is prime to 30 .

The case of unipotent algebraic groups was considered in some detail in [Ch1]- Ch3], [DM], [BM]. However, the following examples show that it may not be easy to combine the two cases. Namely, for a perfect (or even strictly perfect) group $G$ and a word $w=x^{m}$ such that the power map $\widetilde{w}$ is surjective on both $H=G / U$ and $U=R_{u}(G)$, the map $\widetilde{w}$ may or may not be surjective on $G$.

Example 3.1. Let $G=H U$ where $H=\mathrm{SL}_{2}(\mathbb{C})$ and $U$ is the 6-dimensional irreducible $H$-module. Let $m=3$. Then the word map $x \mapsto x^{3}$ is surjective on $H$ and on $U$. The weights of $U$ are $\xi^{5}, \xi^{3}, \xi, \xi^{-1}, \xi^{-3}, \xi^{-5}$ where $\xi: T \rightarrow \mathbb{C}^{*}$ is the weight of the natural 2-dimensional representation (indeed, the 6-dimensional representation is the representation on 5 -forms in 2 -variables). Hence $G$ is a strictly perfect group. Let $\sigma \in H$ be an element of order 3, and let $g$ be any element of $H$ such that $g^{3}=\sigma$. We may assume $g, \sigma \in T$. Then in the basis consisting of the weight vectors the elements $g$ and $\sigma$ are represented by the following diagonal matrices:

$$
g=\operatorname{diag}\left(\epsilon_{9}^{5}, \epsilon_{9}^{3}, \epsilon_{9}, \epsilon_{9}^{-1}, \epsilon_{9}^{-3}, \epsilon_{9}^{-5}\right)
$$

where $\epsilon_{9}=\xi(g)=\sqrt[9]{1}$,

where $\epsilon_{3}=\xi\left(g^{3}\right)=\sqrt[3]{1}$.

$$
\sigma=\operatorname{diag}\left(\epsilon_{3}^{5}, 1, \epsilon_{3}, \epsilon_{3}^{-1}, 1, \epsilon_{3}^{-5}\right)
$$

For every $m$ we have

$$
(g u)^{m}=g^{m} N_{g}(u)
$$

where

$$
N_{g}(u)=\left(g^{1-m} u g^{m-1}\right) \cdots\left(g^{-1} u g\right) u
$$


We may view $N_{g}$ as a linear operator $N_{g}: U \rightarrow U$ which is equal to the sum of linear operators $1+g+$ $g^{2}+\cdots+g^{m-1}$. Thus, for $m=3$ we have

$$
N_{g}=1+g+g^{2} .
$$

Let $v \in U$ be a weight vector corresponding to the weight $\xi^{3}$, then $N_{g}(v)=\left(1+\epsilon_{3}+\epsilon_{3}^{2}\right) v=0$, and therefore there is no $u$ such that $N_{g}(u)=v$. Hence $\sqrt[3]{\sigma v} \notin G$, and therefore the map $x \mapsto x^{3}$ is not surjective on $G$.

Example 3.2. Let $G=H U$ where $H=\mathrm{PSL}_{2}(\mathbb{C})$ and $U$ is the 3-dimensional (adjoint) irreducible $H$-module. Then the word map induced by $w=x^{m}$ is surjective on $H$ and on $U$. Also, the word map $x \mapsto x^{m}$ is surjective on $G$.

Proof. Let $\sigma \in H$, and let $g \in G$ be such that $g^{m}=\sigma$. We may assume $m>1$ and $\sigma \neq 1$. Consider the linear operator $N_{g}=1+g+\cdots+g^{m-1}$ on $U$. We have

$$
N_{g}(u)=0 \text { for some } u \in U \Leftrightarrow \text { the eigenvalues of } g \text { are } \sqrt[m]{1} \Leftrightarrow \sigma=g^{m}=1
$$

(note that both non-identity eigenvalues of $g$ are of the same order). Since $\sigma \neq 1$, the map $N_{g}: U \rightarrow U$ is surjective. Thus, for every $v \in U$ there is $u \in U$ such that $N_{g}(u)=v$. Hence $\sqrt[m]{\sigma v}=g u \in G$, and therefore the map $x \mapsto x^{m}$ is surjective on $G$.

3.2. Commutator word. Ore's problem. In 1951 Oystein Ore proved (see $\mathrm{Or}$ ) that every element $g$ of the alternating group $A_{n}, n \geq 5$, is a single commutator $g=[\sigma, \tau], \sigma, \tau \in A_{n}$. At the end of the paper he wrote: "It is possible that a similar theorem holds for any simple group of finite order, but it seems that at present we do not have the necessary methods to investigate the question."

The conjecture of O. Ore (known now as Ore's problem) was proved only in 2010 by Liebeck, O'Brien, Shalev, Tiep [LOST1. The solution indeed used advanced techniques which were, of course, unavailable in 1951: the classification of finite simple groups, the Deligne-Lusztig theory of characters of finite groups of Lie type, as well as advanced computer algebra. Also, the authors used solution of Ore's problem for groups of Lie type over fields containing more than 8 elements [EG1]. The interested reader is referred to the Bourbaki talk by Malle $\mathrm{Ma}$. for a detailed account of this story.

Thus, now we can say that for every finite simple group $G$ the word map $\widetilde{w}: G \times G \rightarrow G$ induced by $w=[x, y]$ is surjective.

Even before Ore's paper, in 1949, M. Gotô Got proved the surjectivity of the word map $\widetilde{w}: G \times G \rightarrow G$ for $w=[x, y]$ and for a simple compact Lie group $G$, which is the group of real points $G=\mathcal{G}(\mathbb{R})$ of an anisotropic simple algebraic $\mathbb{R}$-group $\mathcal{G}$ (see $[\mathrm{VO}, 5.2]$ ). This result was later on extended to simple complex Lie groups [PW] and, more generally, to $G=\mathcal{G}(K)$ where $\mathcal{G}$ is a simple algebraic group over an algebraically closed field $K$ of arbitrary characteristic [Re]. (Note that recently Gotô's method was applied in a more general context ET, Gor4, see Section 3.4 for some details.)

If $K$ is an infinite field and $\mathcal{G}$ is a simple, simply connected, split $K$-group, then the word map for $w=[x, y]$ is surjective on $G \backslash Z(G)$ (see [EG1]). The same is true for a finite field $K$ and any simply connected group $\mathcal{G}$ [EG1], LOST1] (except for the cases where $G / Z(G)$ is not a finite simple group).

Combining the results mentioned above with a theorem of Blau [B] on representing central elements of finite groups of Lie type as commutators, we may formulate

Theorem C. Let $\mathcal{G}$ be a simple, simply connected algebraic $K$-group, and let $G=\mathcal{G}(K)$. Further, let $\widetilde{w}: G \times G \rightarrow G$ be the word map induced by $w=[x, y]$.

(i) If $K$ is an algebraically closed field or a finite field such that $G / Z(G)$ is a simple group, then $\operatorname{Im} \widetilde{w}=G$.

(ii) If $K=\mathbb{R}$ and $\mathcal{G}$ is an anisotropic group, then $\operatorname{Im} \widetilde{w}=G$.

(iii) If $\mathcal{G}$ is split over $K$, then $\operatorname{Im} \widetilde{w} \supset G \backslash Z(G)$.

Remark D. In (iii) we take $G \backslash Z(G)$ instead of the whole $G$ because elements of the centre $Z(G)$ may not be representable by a single commutator of elements of $G$. Say, let $G=\mathrm{SL}_{2}(\mathbb{R})$. It is well known 
that the matrix -1 is not a commutator. Indeed, if $-1=[x, y]$, then it is easy to see that $x^{2}=y^{2}=-1$, and therefore $\langle x, y\rangle=Q_{8}$ is a quaternion group. But $Q_{8} \nsubseteq \mathrm{SL}_{2}(\mathbb{R})$.

3.3. Commutator width. For any group $G$ the commutator width $l_{c}(G)$ is defined as the smallest $n \in \mathbb{N} \cup \infty$ such that for the word

$$
w=\left[x_{1}, y_{1}\right]\left[x_{2}, y_{2}\right] \cdots\left[x_{n}, y_{n}\right]
$$

the word map $\widetilde{w}: G^{n} \times G^{n} \rightarrow[G, G]$ is surjective.

Example 3.3. Remark D shows that in Theorem $\mathrm{C}\left(\right.$ iii) we may have $l_{c}(G)>1$ where $G=\mathcal{G}(K)$ and $\mathcal{G}$ is a simple, simply connected, split $K$-group. However, every element $\gamma \in Z(G)$ can be represented as a product $g_{1} g_{2}$ where $g_{1}, g_{2} \notin Z(G)$. According to Theorem $\mathrm{C}\left(\right.$ iii), $g_{1}$ and $g_{2}$ are commutators, and therefore $l_{c}(G) \leq 2$.

Example 3.4. Let $A$ be a Noetherian commutative local ring with residue field $K$, let $\mathcal{G}$ be an $A$-group scheme, and let $G=\mathcal{G}(A)$. Suppose that the special fibre $\mathcal{G}_{K}$ is a simple, simply connected, split $K$-group. We have $\mathcal{G}_{K}(K)=G / M$ where $M$ is the congruence subgroup of $G$. If $K$ is big enough (in particular, if $K$ is an infinite field), then every $g \in G, g \notin Z(G) M$ is a single commutator [GS, Theorem 3]. The same arguments as in the previous example show that $l_{c}(G) \leq 2$.

Proposition 3.5. Let $G$ be a firm perfect group over a field $K$ of characteristic zero. Then $l_{c}(G) \leq 2$.

Proof. It follows from Theorem 2.15.

Remark 3.6. In the case where $G$ is a finite perfect group, examples with $l_{c}(G)>1$ are known long ago (see, e.g., Is]). Recently, sharp estimates for $l_{c}(G)$ have been obtained for quasi-simple groups LOST2]. However, for arbitrary finite perfect groups one cannot hope for general estimates: the commutator width is unbounded, see $\mathrm{HP}$, $\mathrm{Ni}$. See [Se, Li] for a survey of results on width with respect to more general words.

3.4. Coxeter elements and Engel words. Coxeter elements were used by Gotô Got for proving the surjectivity of commutator maps. Recently, this approach was applied in [ET] and later on in [Gor4] for studying Engel words. See [EG2] for other applications.

Let $R$ be an irreducible root system. Fix a set of simple roots $\Pi=\left\{\alpha_{1}, \ldots, \alpha_{n}\right\} \subset R$. Let $W$ be the Weyl group of $R$. Any product of reflections $w_{c}=\prod_{i} w_{\alpha_{i}}$ where each $\alpha_{i} \in \Pi$ appears exactly once is called a Coxeter element of $W$ (it is allowed to take reflections $w_{\alpha_{i}}$ in such a product in any order).

Let $\mathcal{G}$ be a simple algebraic $K$-group corresponding to the root system $R$ (here $K$ is not necessarily algebraically closed), and let $\mathcal{T}$ be a maximal torus of $\mathcal{G}$. Let $\mathcal{N}_{\mathcal{G}}(\mathcal{T})$ be the normalizer of $\mathcal{T}$ in $\mathcal{G}$, then $\mathcal{N}_{\mathcal{G}}(\mathcal{T}) / \mathcal{T} \approx W$. For any $w \in W$ let $\dot{w}$ be a fixed preimage in $\mathcal{N}_{\mathcal{G}}(\mathcal{T})$. A Coxeter element $w_{c}$ acts on the Euclidian vector space generated by the simple roots from $\Pi$ without fixed nonzero vectors (see Bou, Planches $\mathrm{I}-\mathrm{X}]$ ). Thus, any preimage $\dot{w}_{c} \in \mathcal{N}_{\mathcal{G}}(\mathcal{T})$ commutes only with the centre of $\mathcal{G}$. Hence the homomorphism $\left[\dot{w}_{c}, *\right]: \mathcal{T} \rightarrow \mathcal{T}, t \mapsto\left[w_{c}, t\right]$, has a finite kernel and is therefore surjective (as a map on the algebraic group $\mathcal{T}$ but not necessarily on $\mathcal{T}(K))$. Hence the map

$$
\begin{gathered}
\Phi_{c}^{m}=\underbrace{\left[\dot{w}_{c},\left[\dot{w}_{c}, \cdots\left[\dot{w}_{c}, *\right] \cdots\right]\right]}_{m-\text { times }}: \mathcal{T} \rightarrow \mathcal{T}, \\
t \mapsto\left[\dot{w}_{c},\left[\dot{w}_{c}, \cdots\left[\dot{w}_{c}, t\right]\right]\right]
\end{gathered}
$$

is surjective for any $m \geq 1$.

Now let $\mathcal{T}$ be a $K$-torus. Denote $T=\mathcal{T}(K)$, then $N_{G}(T)=\mathcal{N}_{\mathcal{G}}(\mathcal{T})(K)$.

Proposition 3.7. Suppose that the following conditions hold:

(a) $\dot{w}_{c} \in N_{G}(T)$ for some Coxeter element $w_{c} \in W$;

(b) the group $T$ is divisible. 
Then the map

$$
\varphi_{c}^{m}=\left[\dot{w}_{c},\left[\dot{w}_{c}, \cdots\left[\dot{w}_{c}, *\right] \cdots\right]\right]: T \rightarrow T
$$

is surjective.

Proof. The map $\varphi_{c}^{m}$ is a homomorphism of the infinite divisible abelian group $T$ with finite kernel.

Corollary 3.8. Suppose that one of the following conditions holds:

(i) the torus $\mathcal{T}$ is completely split and $K^{*}$ is a divisible group;

(ii) $K=\mathbb{R}$ is the field of real numbers and $\mathcal{G}$ is anisotropic.

Then the map

is surjective.

$$
\varphi_{c}^{m}=\left[\dot{w}_{c},\left[\dot{w}_{c}, \cdots\left[\dot{w}_{c}, *\right] \cdots\right]\right]: T \rightarrow T
$$

Proof. Condition (i) contains condition (b) of Proposition 3.7 and condition (b) obviously holds.

If $\mathcal{G}$ is an anisotropic $\mathbb{R}$-group, then $G=\mathcal{G}(\mathbb{R})$ is a connected compact Lie group. Hence $N_{G}(T) / T \approx W$ GG, 6.9.6]. Hence we have $\dot{w}_{c} \in N_{G}(T)$. Note that $T=S^{1} \times S^{1} \times \cdots \times S^{1}$ where $S^{1}$ is the one-dimensional anisotropic $\mathbb{R}$-torus. Hence $T$ is a divisible group.

Now let

be an Engel word.

$$
w_{E}^{m}=[y,[y, \cdots[y, x] \cdots]]
$$

Corollary 3.9. Suppose that the torus $\mathcal{T}$ is completely split and $K^{*}$ is a divisible group. Then for any Engel word $w_{E}^{m}$ the image $\operatorname{Im} \widetilde{w}_{E}^{m}$ of the word $\widetilde{w}_{E}^{m}: G \times G \rightarrow G$ contains all semisimple elements and all unipotent elements.

Proof. The statement for semisimple elements immediately follows from Corollary $3.8(\mathrm{i})$.

Since $\mathcal{G}$ is a split group, all unipotent elements of $G$ are conjugate to an element of a fixed maximal connected unipotent subgroup $U \leq G$, which is normalized by the corresponding group $T$. Since $K^{*}$ is a divisible group, the field $K$ is infinite and therefore there is a regular semisimple element $t \in T$. Then $\widetilde{w}_{E}^{m}(t, U)=U$ (this follows from the well-known equality $[t, U]=U$ which in its turn can be proved by induction on the nilpotency class of $U$ ). So all unipotent elements of $G$ lie in $\operatorname{Im} \widetilde{w}_{E}^{m}$.

The following fact has been proved in ET] for the unitary groups and in [Gor4] for the general case.

Corollary 3.10. Suppose that $K=\mathbb{R}$ is the field of real numbers and $\mathcal{G}$ is anisotropic. Then $\operatorname{Im} \widetilde{w}_{E}^{m}=G$.

Proof. This is a direct consequence of Corollary 3.8 (ii).

Remark 3.11. The question on the surjectivity of Engel words is open. The surjectivity is known for $\mathrm{SL}_{2}$ (see [BZ]), $\mathrm{PGL}_{3}$ (only for $w_{E}^{1}, w_{E}^{2}$ ) and the groups of types $B_{2}, G_{2}$ (see Gor4]). See [BGG] for the surjectivity of Engel words on some finite groups of Lie type.

3.5. Fixed point free elements in the Weyl group. In the previous section we used only one property of Coxeter elements: they act without fixed points on the Euclidian space $\mathcal{E}$ generated by a simple root system $\Pi$. Obviously, the same property is shared by all elements from the conjugacy class $C_{w_{c}}=$ $\left\{w w_{c} w^{-1} \mid w \in W\right\}$ of a Coxeter element $w_{c}$ in the Weyl group (note that all Coxeter elements are conjugate in the Weyl group (see Bou, Ch. 5, Prop. 6.1]) but not every element in this conjugacy class is a Coxeter element). Thus, in Proposition 3.7 and Corollary 3.8 we may replace the preimages of Coxeter elements $\dot{w}_{c}$ with the preimage $\dot{w}$ of any $w \in C_{w_{c}}$.

Below we point out three examples for fixed point free elements in the Weyl group, which can be used in questions like the surjectivity of word maps.

Example 3.12. Any simple group $\mathcal{G}$ contains a semisimple algebraic subgroup $\mathcal{H}=\mathcal{H}_{1} H_{2} \cdots \mathcal{H}_{m}$ such that every simple component $\mathcal{H}_{i}$ of $\mathcal{H}$ is of type $A_{r_{i}}$ and the product of maximal tori $\mathcal{T}_{1} \mathcal{T}_{2} \cdots \mathcal{T}_{m}$ of the components is a maximal torus $\mathcal{T}$ of $\mathcal{G}$ (see [Bo2]). Then in Proposition 3.7 and Corollary 3.8 we may replace the element $\dot{w}_{c}$ with an element of the form $\dot{w}_{1 c} \dot{w}_{2 c} \cdots \dot{w}_{m c}$ where $w_{i c}$ is a Coxeter element of $\mathcal{H}_{i}$. 
Example 3.13. If $\mathcal{G}$ is not of type $A_{r}, D_{2 r+1}$, or $E_{6}$, in Proposition 3.7 and Corollary 3.8 we may also take an element of the form $\dot{w}_{0}$ where $w_{0}$ is the element of maximal weight in $W$ (indeed, in these cases one can check that $\dot{w}_{0}(t)=t^{-1}$ for every $\left.t \in T\right)$.

The following example was used in [HLS].

Example 3.14. Let $R$ be the root system of type $D_{r}$, and let

$$
\alpha_{1}=\epsilon_{1}-\epsilon_{2}, \alpha_{2}=\epsilon_{2}-\epsilon_{3}, \ldots, \alpha_{r-1}=\epsilon_{r-1}-\epsilon_{r}, \alpha_{r}=\epsilon_{r-1}+\epsilon_{r}
$$

be the standard simple root system in the notation of $\mathrm{Bou}$. Put $\beta=\epsilon_{1}-\epsilon_{r}$. Then the element $w^{*}=w_{\beta} w_{\alpha_{r}} w_{\alpha_{r-2}} \cdots w_{\alpha_{2}} w_{\alpha_{1}}$ acts freely on $\mathcal{E}$. Indeed, we may take $\left\{\epsilon_{1}, \ldots, \epsilon_{r}\right\}$ as a basis in $\mathcal{E}$. Then $w^{*}$ acts as a cyclic permutation of the set $\left\{\epsilon_{1}, \ldots, \epsilon_{r},-\epsilon_{1},-\epsilon_{2}, \ldots,-\epsilon_{r}\right\}$, and therefore $w^{*}$ cannot have fixed nonzero vectors in $\mathcal{E}=\left\langle\epsilon_{1}, \ldots, \epsilon_{r}\right\rangle$.

\section{Word MAPS ON $G=\mathrm{SL}_{2}(K)$ AND $G=\mathrm{PGL}_{2}(K)$}

4.1. Semisimple elements. Let $K$ be an algebraically closed field, and let $G=\mathrm{SL}_{2}(K)$. Further, let $1 \neq w \in F_{n}$, and let $\widetilde{w}: \mathrm{SL}_{2}(K)^{n} \rightarrow \mathrm{SL}_{2}(K)$ be the corresponding word map. The first observation here is the following theorem $[\mathrm{BZ}$.

Theorem E. Every semisimple element of $\mathrm{SL}_{2}(K)$ except, possibly, -1 belongs to $\operatorname{Im} \widetilde{w}$.

Proof. Here we give a proof which is a little bit different from [BZ].

Use induction by $n$. If $n=1$, the statement is obvious. Suppose that the statement holds for every $w \in F_{n-1}$. If $w\left(1, x_{2}, \ldots, x_{n}\right)$ is not a trivial word, we may use the induction hypothesis. Thus, we may assume that $w\left(1, x_{2}, \ldots, x_{n}\right)=1$. Further, since $\widetilde{w}$ is dominant, we have $\widetilde{w}\left(g_{1}, \ldots, g_{n}\right) \neq 1$ for some $\left(g_{1}, \ldots, g_{n}\right) \in G^{n}$. We may assume $g_{1} \notin Z(G)$ and

$$
g_{1}=\left(\begin{array}{cc}
1 & a \\
b & 1+a b
\end{array}\right)=\left(\begin{array}{ll}
1 & 0 \\
b & 1
\end{array}\right)\left(\begin{array}{ll}
1 & a \\
0 & 1
\end{array}\right) .
$$

Indeed, every non-central element of $\mathrm{SL}_{2}(K)$ is conjugate to an element of the form (4.1) (see [EG1]), and therefore we may take the $n$-tuple $\left(\sigma g_{1} \sigma^{-1}, \ldots, \sigma g_{n} \sigma^{-1}\right)$ instead of $\left(g_{1}, \ldots, g_{n}\right)$.

Fix these $g_{2}, \ldots, g_{n}$. Let $\Psi: K^{2} \rightarrow K$ be given by

$$
\Psi(x, y)=\operatorname{tr}\left(w\left(\left(\begin{array}{cc}
1 & x \\
y & 1+x y
\end{array}\right), g_{2}, \ldots, g_{n}\right)\right)
$$

(here we take the trace of the matrix $\left.w\left(\left(\begin{array}{cc}1 & x \\ y & 1+x y\end{array}\right), g_{2}, \ldots, g_{n}\right)\right)$. The function $\Psi(x, y)$ is polynomial and $\Psi(0,0)=2$ because $w\left(1, g_{2}, \ldots, g_{n}\right)=1$, and $\Psi(a, b) \neq 2$ because $w\left(g_{1}, \ldots, g_{n}\right) \neq 1$. Thus, $\Psi(x, y)$ is a non-constant polynomial and therefore $\operatorname{Im} \Psi=K$. Formula (4.2) implies that $\operatorname{Im} \Psi \subset \operatorname{Im} \operatorname{tr} \circ \widetilde{w}$. Hence $\operatorname{Im} \operatorname{tr} \circ \widetilde{w}=K$. Thus, for every $\alpha \in K$ there is $g \in \operatorname{Im} \widetilde{w}$ such that $\operatorname{tr} g=\alpha$. If $\alpha \neq \pm 2$, the condition $\operatorname{tr} g=\alpha$ determines a semisimple element $g \in G$ up to conjugacy. If $\operatorname{tr} g= \pm 2$, then $g= \pm u$ where $u$ is a unipotent element. Note that $w(1,1, \ldots, 1)=1$, and therefore $1 \in \operatorname{Im} w$. Hence we have every semisimple element in Im $\widetilde{w}$ except, possibly, -1 .

The following corollary is also contained in $[\mathrm{BZ}]$.

Corollary F. Let $G=\mathrm{PGL}_{2}(K)$, and let $C_{u}$ be the conjugacy class of a non-trivial unipotent element $u \in G$. Further, let $\widetilde{w}: G^{n} \rightarrow G$ be the word map induced by a non-trivial word $w \in F_{n}$. Then Im $w \supset$ $G \backslash C_{u}$.

Remark 4.1. We do not know if $-1 \in \operatorname{Im} \widetilde{w}$ for every word $w$. 
Since every simple algebraic group of Lie rank $r$ (which is not of type $A_{r}, r>1, D_{2 k+1}, k>1$, or $E_{6}$ ) contains a product of $r$ copies of groups of rank one, we also have the following fact, which is a corollary of Theorem E (see [GKP1], GKP2]).

Corollary G. Let $G$ be a simple algebraic group. Suppose that $G$ is not of type $A_{r}, r>1, D_{2 k+1}, k>1$, or $E_{6}$, and let $\widetilde{w}: G^{m} \rightarrow G$ be a non-trivial word map. Then every regular semisimple element of $G$ is contained in $\operatorname{Im} \widetilde{w}$. Moreover, for every semisimple $g \in G$ there exists $g_{0} \in G$ of order $\leq 2$ such that $g g_{0} \in \operatorname{Im} \widetilde{w}$.

4.2. Problem of unipotent elements. For $G=\mathrm{SL}_{2}(K)$, where $K$ is an algebraically closed field, the question whether or not for every word $w \in F_{n}$ the image of the word map $\widetilde{w}: G^{n} \rightarrow G$ contains a non-trivial unipotent element is wide open. This is unknown even in the case $n=2, K=\mathbb{C}$.

This problem is related to the structure of the representation variety

$$
R\left(\Gamma_{w}, \mathrm{SL}_{2}(K)\right)=\left\{\rho: \Gamma \rightarrow \mathrm{SL}_{2}(\mathbb{C})\right\},
$$

where $\Gamma_{w}=F_{n} /\langle w\rangle$ is a group with $n$ generators and one relation $w$. Namely, let

$$
\begin{aligned}
& \mathcal{T}_{w}=\left\{\left(g_{1}, \ldots, g_{n}\right) \in G^{n} \quad \mid \operatorname{tr} \widetilde{w}\left(g_{1}, \ldots, g_{n}\right)=2\right\}, \\
& \mathcal{W}_{w}=\left\{\left(g_{1}, \ldots, g_{n}\right) \in G^{n} \quad \mid \widetilde{w}\left(g_{1}, \ldots, g_{n}\right)=1\right\} .
\end{aligned}
$$

Then $\mathcal{T}_{w}$ is the affine variety of the $n$-tuples $\left(g_{1}, \ldots, g_{n}\right) \in G^{n}$ mapped onto the set of the unipotent elements of $G$, and $\mathcal{W}_{w}$ is the set of the $n$-tuples $\left(g_{1}, \ldots, g_{n}\right) \in G^{n}$ satisfying the relation $w\left(g_{1}, \ldots, g_{n}\right)=1$. Then $\mathcal{W}_{w}=R\left(\Gamma_{w}, \mathrm{SL}_{2}(K)\right)$ (see [LM, page 4]). Note that $\mathcal{W}_{w} \subseteq \mathcal{T}_{w}$ and

$$
\mathcal{W}_{w} \neq \mathcal{T}_{w} \Leftrightarrow \text { there exists a non-trivial unipotent element } u \in \operatorname{Im} w .
$$

Further, let $\mathcal{T}_{w}=\cup_{j} \mathcal{T}_{w}^{j}, \mathcal{W}_{w}=\cup_{i} \mathcal{W}_{w}^{i}$ be the decompositions into irreducible components. Then each $\mathcal{W}_{i}$ is contained in some $\mathcal{T}_{w}^{j}$. Note that $\mathcal{T}_{w}$ is an equidimensional variety of dimension $3 n-1$ (indeed, $\mathcal{T}_{w}$ is a hypersurface in $G^{n}$ and the ring of regular functions on $G^{n}$ is factorial). Suppose that there exists a component $\mathcal{W}_{w}^{i}$ of $\mathcal{W}_{w}$ such that $\operatorname{dim} \mathcal{W}_{w}^{i}<3 n-1$. Then we have $\mathcal{W}_{w}^{i} \varsubsetneqq \mathcal{T}_{w}^{j}$ for some $j$, and therefore $\mathcal{T}_{w}^{j} \backslash \mathcal{W}_{w} \neq \emptyset$ (recall that we consider varieties and therefore we have no embedded components $\left.\mathcal{W}_{w}^{i} \subset \mathcal{W}_{w}^{k}\right)$. Thus we have the implication

the existence of an irreducible component $\mathcal{W}_{w}^{i}$ where $\operatorname{dim} \mathcal{W}_{w}^{i}<3 n-1 \Rightarrow$ the existence a non-trivial unipotent element $u \in \operatorname{Im} \widetilde{w}$.

We do not know whether the converse implication is true.

Question 4.2. Is it true that if $\operatorname{Im} \widetilde{w}$ contains a non-trivial unipotent element $u$, then $\operatorname{dim} \mathcal{W}_{w}^{i}<3 n-1$ for some component $\mathcal{W}_{w}^{i}$ ?

In GKP1], GKP2 the structure of the varieties $\mathcal{W}_{w}, \mathcal{T}_{w}$ was described in the case $n=2, K=\mathbb{C}$ for $w=\left[x^{k}, y^{m}\right], w=[x, y]^{p}$ where $p$ is prime and $w=\left[[x, y], x[x, y] x^{-1}\right]$. In all these cases we have a component of dimension 4 or 3 , which is strictly less than $3 \cdot 2-1=5$, and therefore there is a non-trivial $u \in \operatorname{Im} w$. The investigation of the structure $R\left(\Gamma_{w}, \mathrm{SL}_{2}(\mathbb{C})\right)$ is interesting from other points of view (see, e.g., [LM, Section 7]). Perhaps better understanding of this structure might also give a clue to the problem of the existence of non-trivial unipotent elements in $\operatorname{Im} \widetilde{w}$.

4.3. Magnus embedding. Let $F_{n}=\left\langle x_{1}, \ldots, x_{n}\right\rangle$, and let $A=\mathbb{Z}\left[t_{1}^{ \pm 1}, \ldots, t_{n}^{ \pm 1}, s_{1}, \ldots, s_{n}\right]$ where $t_{j}, s_{i}$ are algebraically independent variables (over $\mathbb{Z})$. The map

$$
x_{i} \rightarrow \zeta_{i}=\left\{\left(\begin{array}{cc}
t_{i} & s_{i} \\
0 & t_{i}^{-1}
\end{array}\right)\right\}
$$

induces the injective homomorphism

$$
F_{n} / F_{n}^{2} \hookrightarrow \Upsilon=\left\{\left(\begin{array}{cc}
\alpha & \beta \\
0 & \alpha^{-1}
\end{array}\right) \mid \alpha \in A^{*}, \beta \in A\right\}
$$

called the Magnus embedding (see $[\mathrm{BZ}]$ ). In $[\mathrm{BZ}$ the following consequence of the Magnus embedding was established: 
Theorem H. Let $L$ be a field of characteristic zero, and let $w \in F_{n} \backslash F_{n}^{2}$. Further, let $G=\mathrm{SL}_{2}(L)$, and let $\widetilde{w}: G^{n} \rightarrow G$ be the corresponding word map. Then the set $\operatorname{Im} \widetilde{w}$ contains all unipotent elements.

Using the Morozov-Jacobson Theorem, one can extend Theorem $\mathrm{H}$ to groups $G=\mathcal{G}(L)$ where $\mathcal{G}$ is any semisimple group defined over $L$, see [BZ].

Also, in BZ there is the following corollary of Theorem $\mathrm{H}$.

Corollary I. Let $G=\mathrm{PGL}_{2}(K)$ where $K$ is an algebraically closed field of characteristic zero, and let $w \notin F_{n}^{2}$. Then the word map $\widetilde{w}: G^{n} \rightarrow G$ is surjective.

One cannot extend this result to the case of characteristic $p>0$. For instance, if $\operatorname{char} K=p>0$ and $w=x^{p}$, then there are no non-trivial unipotent elements in $\operatorname{Im} \widetilde{w}$. However, we may give the following generalization of Theorem $\mathrm{H}$ and Corollary I.

Theorem 4.3. Let $L$ be an infinite field, and let $w \in F_{n} \backslash F_{n}^{2}$. Further, let $G=\operatorname{SL}_{2}(L)$, and let $\widetilde{w}: G^{n} \rightarrow G$ be the corresponding word map. Then there exists a finite set of primes $S_{w}$ such that if $p=\operatorname{char} L \notin S_{w}$ (in particular, if $p=0$ ), then the set $\operatorname{Im} \widetilde{w}$ contains a non-trivial unipotent element.

Proof. We may assume $w \in F_{n}^{1} \backslash F_{n}^{2}$ (for the case $w \notin F^{1}$ the result is easily reduced to the case $w=x^{m}$ where the inclusion $u \in \operatorname{Im} \widetilde{w}$ is obvious for the cases where $p \nmid m)$.

Also, denote by the same symbol $\widetilde{w}$ the corresponding word map $\mathrm{SL}_{2}(A)^{n} \rightarrow \mathrm{SL}_{2}(A)$ (recall that $A=\mathbb{Z}\left[t_{1}^{ \pm 1}, \ldots, t_{n}^{ \pm 1}, s_{1}, \ldots, s_{n}\right]$ ). Consider the restriction of $\widetilde{w}$ to $\Upsilon$ (see (4.4)): Res $\Upsilon \widetilde{w}: \Upsilon^{n} \rightarrow \Upsilon$. Since $w \in F_{n}^{1} \backslash F_{n}^{2}$, the Magnus embedding (see (4.4)) implies

$$
\widetilde{w}\left(\zeta_{1}, \ldots, \zeta_{n}\right)=\left(\begin{array}{cc}
1 & f_{w} \\
0 & 1
\end{array}\right) \neq\left(\begin{array}{cc}
1 & 0 \\
0 & 1
\end{array}\right)
$$

where $0 \neq f_{w}=f_{w}\left(t_{1}^{ \pm 1}, \ldots, t_{n}^{ \pm 1}, s_{1}, \ldots, s_{n}\right) \in A$. Put

$$
S_{w}=\text { all common prime divisors of all coefficients of } f_{w}\left(t_{1}^{ \pm 1}, \ldots, t_{n}^{ \pm 1}, s_{1}, \ldots, s_{n}\right) \text {. }
$$

Let $p=\operatorname{char} L \notin S_{w}$. For every $a \in A$ denote by $\bar{a}$ the image of $a$ with respect to the natural homomorphism $A \rightarrow A /(p)=(\mathbb{Z} / p \mathbb{Z})\left[t_{1}^{ \pm 1}, \ldots, t_{n}^{ \pm 1}, s_{1}, \ldots, s_{n}\right]$. Since $p \notin S_{w}$, we have $\bar{f}_{w} \neq 0$. The matrices $\zeta_{i}$ may be viewed as matrices over $A /(p)$ because their entries are variables $t_{i}^{ \pm 1}, s_{i}$ (see (4.3)). Thus, we have the matrix equality over the $\operatorname{ring} A /(p)$ :

$$
\widetilde{w}\left(\zeta_{1}, \ldots, \zeta_{n}\right)=\left(\begin{array}{cc}
1 & \bar{f}_{w} \\
0 & 1
\end{array}\right) \neq\left(\begin{array}{ll}
1 & 0 \\
0 & 1
\end{array}\right) .
$$

Since $L$ is an infinite field, the Laurent polynomial $\bar{f}_{\omega}\left(t_{1}, \ldots, t_{n}, s_{1}, \ldots, s_{n}\right)$ is not identically zero on $\left(L^{*}\right)^{n} \times L^{n}$. Therefore there is $\left(\alpha_{1}, \ldots, \alpha_{n}, \beta_{1}, \ldots, \beta_{n}\right) \in(L *)^{n} \times L^{n}$ such that $\bar{f}_{\omega}\left(\alpha_{1}, \ldots, \alpha_{n}, \beta_{1}, \ldots, \beta_{n}\right) \neq$ 0 . Now we get our statement from (4.5) applying the substitutions

$$
\zeta_{i} \rightarrow\left(\begin{array}{cc}
\alpha_{i} & \beta_{i} \\
0 & \alpha_{i}^{-1}
\end{array}\right)
$$

Together with Theorem E, this gives the following corollary.

Corollary 4.4. Let $G=\mathrm{PGL}_{2}(K)$ where $K$ is an algebraically closed field, and let $w \notin F_{n}^{2}$. Then there exists a finite set of primes $S_{w}$ such that if $p=$ char $K \notin S_{w}$ (in particular, if $p=0$ ), then the word map $\widetilde{w}: G^{n} \rightarrow G$ is surjective. 


\section{WORD MAPS WITH CONSTANTS}

Studying word maps, we are naturally led to extending the set-up by considering words with constants, see Gor2, GKP1, GKP2. (As a vague analogy, one can think of investigating any functions in $n$ variables and then considering substitutions of constants instead of some variables.)

Let $G$ be a group, let $\Sigma=\left(\sigma_{1}, \ldots, \sigma_{r}\right)$ where $\sigma_{i} \in G \backslash Z(G)$ for every $i=1, \ldots, r$, and let $w_{1}, \ldots, w_{r+1} \in$ $F_{n}$. The expression $w_{\Sigma}=w_{1} \sigma_{1} w_{2} \sigma_{2} \cdots w_{r} \sigma_{r} w_{r+1}$ is called $a$ word with constants (or a generalized monomial) if the sequence $w_{2}, \ldots, w_{r}$ does not contain the identity word.

Equivalently, one can think of a word with constants as of an element of the free product $G * F_{n}$, see, e.g., $\mathrm{KT}$.

We will view a word $w \in F_{n}$ as a word with constants $w_{\Sigma}$ with $\Sigma=\emptyset$ and $w=w_{1}$. A word with constants also induces a map $\widetilde{w}_{\Sigma}: G^{n} \rightarrow G$.

If $G=\mathcal{G}(K)$ where $\mathcal{G}$ is a semisimple algebraic group, then $\operatorname{Im}_{\widetilde{w}_{\Sigma}}$ is not necessarily Zariski dense in $\mathcal{G}$ as in Borel's Theorem.

Example 5.1. Let $w_{\Sigma}$ be a word with constants $\Sigma=\left(\sigma_{1}, \ldots, \sigma_{r}\right)$, and let $\tau \in \mathcal{G}$. Further, let $w_{\Sigma^{\prime}}^{\prime}=$ $w_{\Sigma} \tau w_{\Sigma}^{-1}$. Then $\operatorname{Im} \widetilde{w}_{\Sigma^{\prime}}^{\prime}$ is contained in the conjugacy class of $\tau$, and therefore the map $\widetilde{w}_{\Sigma^{\prime}}^{\prime}$ cannot be dominant. The same refers to the word map for the word $w_{\Sigma^{\prime \prime}}^{\prime \prime}=\left[w_{\Sigma}, \tau\right]$, where the image is equal to $\left(\operatorname{Im} \widetilde{w}_{\Sigma^{\prime}}^{\prime}\right) \tau^{-1}$.

Consider some problems related to words with constants.

5.1. Covering number. Consider the word with constants $w_{\Sigma}=x_{1} \sigma_{1} x_{1}^{-1} x_{2} \sigma_{2} x_{2}^{-1} \cdots x_{m} \sigma_{m} x_{m}^{-1}$ and the word map $\widetilde{w}_{\Sigma}: G^{m} \rightarrow G$. Then $\operatorname{Im} \widetilde{w}_{\Sigma}=C_{1} C_{2} \cdots C_{m}$ where $C_{i}$ is the conjugacy class of $\sigma_{i}$. The minimal number $d \in \mathbb{N}$ such that $\operatorname{Im} \widetilde{w}_{\Sigma}=G$ for every $\Sigma=\left(\sigma_{1}, \ldots, \sigma_{m}\right)$ with $m>d$ is called the extended covering number of $G$; it is called the covering number under the additional condition $\sigma_{1}=\sigma_{2}=\cdots=\sigma_{m}$.

If $G=\mathcal{G}(K)$ where $\mathcal{G}$ is a simple algebraic $K$-group, then $\widetilde{w}_{\Sigma}$ is dominant for $m \geq 2 \operatorname{rank} G+1$ (see [Gor1]), and therefore it is surjective for $m \geq 4 \operatorname{rank}(G)+2$. We do not discuss here numerous computations of precise values of covering numbers for different types of algebraic groups as well as particular cases where the set $\Sigma$ consists of special elements, reflections, root subgroups, etc. It is worth mentioning the general result by Nikolov [Ni] saying that the extended covering number of $G$ can be arbitrarily large when $G$ runs over all finite groups of Lie type.

5.2. Thompson's Conjecture. Thompson's Conjecture asserts that any finite simple group $G$ contains a conjugacy class $C$ such that $C^{2}=G$. The conjecture has been proved for $A_{n}, n \geq 5$, the sporadic groups and the simple groups of Lie type over fields containing more than 8 elements (see [EG1 and the references therein). The existence of such a conjugacy class has also been proved for the cases $G=\mathcal{G}(K) / Z(\mathcal{G}(K))$ where $\mathcal{G}$ is a split, simple, simply connected algebraic group over an infinite field $K$ or a simple anisotropic group over $K=\mathbb{R}$ (a compact Lie group) (see [EG1], Gor1, [ET]).

Note that the Thompson conjecture is the question on the surjectivity of word maps with constants induced by $w_{\Sigma}=x \sigma x^{-1} y \sigma y^{-1}$ (here $\Sigma=\{\sigma\}$ ).

5.3. Identities with constants. For a simple algebraic group $G$ it may happen that a word map with constants $\widetilde{w}_{\Sigma}: G^{n} \rightarrow G$ is trivial (that is, $\operatorname{Im} \widetilde{w}_{\Sigma}=\{1\}$ ) for a non-trivial word with constants $w_{\Sigma}$. Such a word $w_{\Sigma}$ is called an identity with constants. Identities with constants were studied, in particular, in GM], To, Gor2, Step1, [Step2. Interestingly, they play an important role in description of some "big" subgroups of $G$ (see [Step1], Step2]).

A simple group $G$ has identities with constants if and only if the corresponding root system $R$ contains roots of different lengths. Moreover, the constants are special "small" elements Gor2]. For any simple group with roots of different lengths there is, in any case, at least one example of an identity with constants Gor2, Step1. However, there is no description of all such identities. 
5.4. Dimension of the image of general word maps with constants. Throughout this and the next section we assume that the ground field is algebraically closed. One of the first questions to ask when describing word maps with constants is the question on the dimension of the image. Let $w_{\Sigma}=$ $w_{1} \sigma_{1} w_{2} \sigma_{2} \cdots w_{r} \sigma_{r} w_{r+1}$ be a word with constants where $w_{i}=w_{i}\left(x_{1}, \ldots, x_{n}\right) \in F_{n}$ and $\Sigma=\left(\sigma_{1}, \ldots, \sigma_{r}\right)$ is an $r$-tuple of elements of a semisimple algebraic group $G$. In GKP2 we proved that there exists an open subset $U \subset G^{r}$ such that for all $\Sigma \in U$ the word maps with constants $\widetilde{w}_{\Sigma}: G^{n} \rightarrow G$ have the images of the same dimension $\mathfrak{d}$.

We will not strictly follow the definition of a word map and admit that every $\Sigma \in G^{r}$ induces the word map with constants $\widetilde{w}_{\Sigma}$. (In fact, if $\Sigma$ contains elements from the centre of $G$, we may have $w_{\Sigma}=1$. But in such a case we put $w_{\Sigma}\left(g_{1}, \ldots, g_{n}\right)=1$ for every $\left(g_{1}, \ldots, g_{n}\right) \in G^{n}$ by definition.) Then for every $\Sigma \in G^{r}$ we have

$$
\operatorname{dim} \operatorname{Im} \widetilde{w}_{\Sigma} \leq \mathfrak{d}
$$

(see GKP2]). Thus, for given words $w_{1}, \ldots, w_{r+1}$ there is a "general" dimension of the image of the word map with constants $w_{\Sigma}=w_{1} \sigma_{1} w_{2} \sigma_{2} \cdots w_{r} \sigma_{r} w_{r+1}$, which is maximal among all possible dimensions.

We may consider the word

$$
w^{Y}=w^{Y}\left(x_{1}, \ldots, x_{n}, y_{1}, \ldots, y_{r}\right)=w_{1} y_{1}^{k_{1}} w_{2} y_{2}^{k_{2}} w_{3} \cdots w_{r} y_{r}^{k_{r}} w_{r+1} \in F_{n+r}
$$

such that the word with constants $w_{\Sigma}=w^{Y}\left(x_{1}, \ldots, x_{n}, \sigma_{1}, \ldots \sigma_{r}\right)$ is obtained from $w^{Y}$ by substituting the $r$-tuple $\Sigma$ instead of $\left(y_{1}, \ldots, y_{r}\right)$. In such a case we can generalize the result mentioned above to any word $\omega\left(x_{1}, \ldots, x_{n}, y_{1}, \ldots, y_{r}\right) \in F_{n+r}$ instead of the words of the special form $w^{Y}$.

Theorem 5.2. Let $G$ be a simple algebraic group. Let $\omega=\omega\left(x_{1}, \ldots, x_{n}, y_{1}, \ldots, y_{r}\right) \in F_{n+r}$ be any nontrivial word in $n+r$ variables. Then there exists an open subset $U \subset G^{r}$ such that for all $\Sigma \in U$ the word maps with constants $\widetilde{w}_{\Sigma}: G^{n} \rightarrow G$ corresponding to the word with constants $w_{\Sigma}=\omega\left(x_{1}, \ldots, x_{n}, \sigma_{1}, \ldots, \sigma_{r}\right)$ have the images of the same dimension $\mathfrak{d}$. Moreover, for every $\Sigma \in G^{r}$ we have

$$
\operatorname{dim} \operatorname{Im} \widetilde{w}_{\Sigma} \leq \mathfrak{d} .
$$

Proof. The proof is almost the same as in [GKP2, Theorem 1.1].

We have dominant maps

$$
\widetilde{\omega}: G^{n+r} \rightarrow G \text { and } p_{Y}: G^{n+r} \rightarrow G^{r}
$$

where $p_{Y}$ is the projection onto the components $n+1, \ldots, n+r$. Consider the map

$$
F: G^{n+r} \stackrel{\left(\widetilde{\omega}, p_{Y}\right)}{\rightarrow} G \times G^{r} .
$$

Let $X=\overline{\operatorname{Im} F} \subset G \times G^{r}$, and let $p_{Y}^{\prime}: X \rightarrow G^{r}$ be the projection onto $G^{r}$. Then $p_{Y}^{\prime}(X)=G^{r}$. Indeed, for every $r$-tuple $\Sigma=\left(\sigma_{1}, \ldots, \sigma_{r}\right) \in G^{r}$ there is a non-empty set

$$
Z_{\Sigma}=\left\{\left(\widetilde{w}_{\Sigma}\left(g_{1}, \ldots, g_{n}\right), \sigma_{1}, \ldots, \sigma_{r}\right) \mid\left(g_{1}, \ldots, g_{n}\right) \in G^{n}\right\} \subset X .
$$

There exists an open subset $\mathcal{V}$ of $X$ such that:

(a) $\mathcal{V} \subset \operatorname{Im} F$,

(b) for every $v \in \mathcal{V}$ the dimension of every irreducible component of the preimage $F^{-1}(v)$ is a fixed number $\mathfrak{f}$,

(c) for every $u \in \operatorname{Im} F$ the dimension of every irreducible component of the preimage $F^{-1}(u)$ is greater than or equal to $\mathfrak{f}$.

Let now $\mathcal{U} \subset G^{r}$ be an open subset contained in $p_{Y}^{\prime}(\mathcal{V})$, and let $\Sigma=\left(\sigma_{1}, \ldots, \sigma_{r}\right) \in \mathcal{U}$. Let $v \in \mathcal{V}$ be such that $p_{Y}^{\prime}(v)=\Sigma$. Then $v=\left(\widetilde{w}_{\Sigma}\left(g_{1}, \ldots, g_{n}\right), \sigma_{1}, \ldots, \sigma_{r}\right)$ for some $\left(g_{1}, \ldots, g_{n}\right) \in G^{n}$, and the dimension of every irreducible component of the preimage $F^{-1}(v)$ is equal to $\mathfrak{f}$, see (b). Further, the Zariski closure $\bar{Z}_{\Sigma}$ is an irreducible closed subset of $X$. Indeed, $Z_{\Sigma}$ is the image of an irreducible variety under the morphism $F_{\Sigma}: G^{n} \rightarrow G \times G^{r}$ given by the formula $F_{\Sigma}\left(x_{1}, \ldots, x_{n}\right)=\left(w_{\Sigma}\left(x_{1}, \ldots, x_{n}\right), \sigma_{1}, \ldots, \sigma_{r}\right)$. Note that $v \in Z_{\Sigma} \cap \mathcal{V}$. Hence there is an open subset $\mathcal{W}$ of $\bar{Z}_{\Sigma}$ such that $v \in \mathcal{W} \subset \mathcal{V}$. Since $\mathcal{W} \subset \mathcal{V}$, the dimension of every irreducible component of $F^{-1}\left(v^{\prime}\right)$ for every point $v^{\prime} \in \mathcal{W}$ is equal to $\mathfrak{f}$, see (b). Also, for every $v^{\prime} \in \mathcal{W}$ the closed subset $F^{-1}\left(v^{\prime}\right) \subset G^{n} \times G^{r}$ is isomorphic (as an affine variety) to the closed 
subset $F_{\Sigma}^{-1}\left(v^{\prime}\right) \subset G^{n}$. Hence the dimension of the general fibre of the morphism $F_{\Sigma}: G^{n} \rightarrow Z_{\Sigma} \subset G \times G^{r}$ is equal to $\mathfrak{f}$, and therefore

$$
\operatorname{dim} \overline{\operatorname{Im} F_{\Sigma}}=n \operatorname{dim} G-\mathfrak{f} .
$$

The construction of $F_{\Sigma}$ shows that $\overline{\operatorname{Im} F_{\Sigma}}$ is isomorphic to $\overline{\operatorname{Im} \widetilde{w}_{\Sigma}}$ (the projection of $G \times G^{r}$ onto the first component gives this isomorphism). Hence $\operatorname{dim} \overline{\operatorname{Im} \widetilde{w}_{\Sigma}}=n \operatorname{dim} G-\mathfrak{f}$ for every $\Sigma \in \mathcal{U}$.

Let $\Sigma^{\prime}=\left(\sigma_{1}^{\prime}, \ldots, \sigma_{r}^{\prime}\right) \in G^{r}$ (possibly, $\sigma_{i}^{\prime} \in Z(G)$ for some $i$ ). The maps $\widetilde{w}_{\Sigma^{\prime}}: G^{n} \rightarrow G, F_{\Sigma^{\prime}}: G^{n} \rightarrow$ $G \times G^{r}$ have the same fibres. Moreover, these fibres are also isomorphic to the fibres of the map $F: G^{n} \times$ $G^{r} \rightarrow G \times G^{r}$ which correspond to points of the form $\left(\widetilde{w}_{\Sigma^{\prime}}\left(g_{1}, \ldots, g_{n}\right), \sigma_{1}^{\prime}, \ldots, \sigma_{r}^{\prime}\right)$. Since the dimension of every fibre of $F$ is at least $\mathfrak{f}$ (see (c)), the dimension $\mathfrak{f}^{\prime}$ of the general fibre of $\widetilde{w}_{\Sigma^{\prime}}$ is at least $\mathfrak{f}$. Hence

$$
\operatorname{dim} \overline{\operatorname{Im} \widetilde{w}_{\Sigma^{\prime}}}=n \operatorname{dim} G-\mathfrak{f}^{\prime} \leq \mathfrak{d}=n \operatorname{dim} G-\mathfrak{f} .
$$

The following Corollary is a strengthening of GKP2, Corollary 1.4].

Corollary 5.3. Let $\omega=\omega\left(x_{1}, \ldots, x_{n}, y_{1}, \ldots, y_{r}\right) \in F_{n+r}$ be any non-trivial word in $n+r$ variables. If $\omega\left(x_{1}, \ldots, x_{n}, 1, \ldots, 1\right) \neq 1$, then there exists an open subset $U \subset G^{r}$ such that for every $\Sigma \in U$ the word map with constants $\widetilde{w}_{\Sigma}: G^{n} \rightarrow G$ corresponding to $w_{\Sigma}=\omega\left(x_{1}, \ldots, x_{n}, \sigma_{1}, \ldots, \sigma_{r}\right)$ is dominant.

Proof. Indeed, for $\Sigma_{0}=(1, \ldots, 1) \in \mathcal{G}^{r}$ the map $\widetilde{w}_{\Sigma_{0}}: \mathcal{G}^{n} \rightarrow \mathcal{G}$ is dominant according to the Borel Theorem. Hence for a general map $\widetilde{w}_{\Sigma}: \mathcal{G}^{n} \rightarrow \mathcal{G}$, by Theorem 5.2 we have $\operatorname{dim} \overline{\operatorname{Im} \widetilde{w}_{\Sigma}} \geq \operatorname{dim} \overline{\operatorname{Im} \widetilde{w}_{\Sigma_{0}}}=$ $\operatorname{dim} G$.

5.5. Word maps with constants and the quotient map. Let $G$ be a semisimple algebraic group, let $T$ be a maximal torus of $G$, and let $W$ be the Weyl group of $G$. Then there is the quotient map

$$
\pi: G \rightarrow T / W
$$

(see [SS, 3.1]) taking every $g \in G$ to the class of its semisimple part $g_{s}$ in $T / W$ (namely, if $g=g_{s} g_{u}$ is the Jordan decomposition and $t=x g_{s} x^{-1} \in T$ is conjugate to $g_{s}$, then $\pi(g)=\bar{t}$ where $\bar{t}$ is the class of $t$ in $T / W)$.

Let now $\widetilde{w}_{\Sigma}: G^{n} \rightarrow G$ be a word map with constants. Consider the composition $\pi \circ \widetilde{w}_{\Sigma}: G^{n} \rightarrow T / W$. If this map is dominant, then so is the word map with constants $\widetilde{w}_{\Sigma}^{\prime}: G^{n+1} \rightarrow G$ corresponding to $w_{\Sigma}^{\prime}=y w_{\Sigma} y^{-1}$. Indeed, $y$ here is a new variable, and therefore $\operatorname{Im} w_{\Sigma}^{\prime}$ contains all elements in $G$ which are conjugate to elements from $\operatorname{Im} w_{\Sigma}$. Since $\pi \circ \widetilde{w}_{\Sigma}$ is dominant, the set $\operatorname{Im} w_{\Sigma}^{\prime}$ contains an open subset of regular semisimple elements of $G$ and is therefore dense in $G$. Thus, if the map $\pi \circ \widetilde{w}_{\Sigma}$ is dominant, the map $\widetilde{w}_{\Sigma}$ is "dominant up to conjugacy", that is, almost all conjugacy classes of $G$ (except for some closed subset of $G$ ) intersect $\operatorname{Im} \widetilde{w}$.

In Theorem 5.2 we have the condition $\omega\left(x_{1}, \ldots, x_{n}, 1, \ldots, 1\right) \neq 1$ for getting a dominant map

$$
\widetilde{\omega}\left(x_{1}, \ldots, x_{n}, \sigma_{1}, \ldots, \sigma_{r}\right): G^{n} \rightarrow G
$$

for a general $\Sigma=\left(\sigma_{1}, \ldots, \sigma_{r}\right) \in G^{r}$. If we drop the condition $\omega\left(x_{1}, \ldots, x_{n}, 1, \ldots, 1\right) \neq 1$, we cannot expect the dominance of the corresponding word map with constants. However, we may hope for the following dichotomy for any word $\omega\left(x_{1}, \ldots, x_{n}, y_{1}, \ldots, y_{r}\right) \in F_{n+r}$ :

$$
\operatorname{Im} \pi \circ \widetilde{\omega}\left(x_{1}, \ldots, x_{n}, \sigma_{1}, \ldots, \sigma_{r}\right)=\left\{\begin{array}{l}
\text { either just one point for every } \\
\Sigma=\left(\sigma_{1}, \ldots, \sigma_{r}\right) \in G^{r}, \\
\text { or } \\
\text { a dense subset in } T / W \text { for } \\
\text { every } \Sigma=\left(\sigma_{1}, \ldots, \sigma_{r}\right) \in U \\
\text { from some open set } U \in G^{r} .
\end{array}\right.
$$

Perhaps such a dihotomy could be the best possible replacement of Borel's theorem in the context of word maps with constants. 
Remark 5.4. Dichotomy ( $)$ definitely holds if $\operatorname{rank} G=1$. Indeed, in this case $\operatorname{dim} T / W=1$, and therefore the image of the irreducible variety $G^{n}$ with respect to the morphism $\pi \circ \widetilde{\omega}\left(x_{1}, \ldots, x_{n}, \sigma_{1}, \ldots, \sigma_{r}\right)$ is either just one point, or a dense subset. We cannot drop the restriction that the second alternative in (\$) holds only for an open subset. Indeed, consider the word $\omega\left(x_{1}, x_{2}, y_{1}, y_{2}\right)=x_{1} y_{1} x_{1}^{-1} x_{2} y_{2} x_{2}^{-1}$. If $\Sigma=\left(\sigma_{1}, \sigma_{2}\right)$ is the set of "small" elements (say, root subgroup elements $x_{\alpha}(s)$ ), then the image of $\widetilde{\omega}\left(x_{1}, x_{2}, \sigma_{1}, \sigma_{2}\right)$ is the product of the conjugacy classes $C_{\sigma_{1}} C_{\sigma_{2}}$, each of which has "small" dimension. Therefore, if $\operatorname{dim} G>\operatorname{dim} \overline{C_{\sigma_{1}} C_{\sigma_{2}}}$, we have $\overline{\operatorname{Im} \pi \circ \widetilde{\omega}\left(x_{1}, x_{2}, \sigma_{1}, \sigma_{2}\right)}<\operatorname{dim} T / W$.

Remark 5.5. For a word $\omega\left(x_{1}, \ldots, x_{n}, y\right)$ it has been proved in GKP2 that for $\Sigma=\sigma \in U$ from some open subset $U \subset G$ the map $\pi \circ \widetilde{\omega}\left(x_{1}, \ldots, x_{n}, \sigma\right): G^{n} \rightarrow T / W$ is dominant under the condition $\widetilde{\omega}(1, \ldots, 1, y)=1$. However, the condition $\widetilde{\omega}(1, \ldots, 1, y)=1$ is rather strong and is unknown even for the case where the set of constants $\Sigma$ is just one element.

Example 5.6. Let $K$ be an algebraically closed field of characteristic zero and let $G=\mathcal{G}(K)$ where $\mathcal{G}$ is a simple adjoint $K$-group. Consider $\omega(x, y)=x^{a} y^{b} x^{c} y^{d}, d \neq-b$ and assume for simplicity that $a$ is prime to 30. We have $\omega(1, \sigma)=\sigma^{b+d} \neq 1$ for almost all $\sigma \in G$. But the word map with constants $\pi \circ \widetilde{\omega}(x, \sigma): G \rightarrow T / W$ is dominant for elements $\sigma$ from some open subset of $G$. Indeed, we may assume $c=-a$ (otherwise we may use Theorem [5.2). Thus we have the word with constants $\left(x^{a} \sigma^{b} x^{-a}\right) \sigma^{d}$. There is an open subset $U \subset G$ such that if $\sigma \in U$, then $\sigma^{b}, \sigma^{d}$ are regular semisimple elements of $G$. Let $C_{\sigma^{b}}$, $C_{\sigma^{d}}$ be the conjugacy classes of $\sigma^{b}, \sigma^{d}$. Then $G \backslash Z(G) \subset C_{\sigma^{b}} C_{\sigma^{d}}$, and therefore $\pi\left(C_{\sigma^{b}} \sigma^{d}\right)$ is a dense subset in $T / W$. On the other hand, the assumption on $a$ implies, by Theorem D., that for every $h \in G$ there is $g \in G$ such that $g^{a}=h$, so that $C_{\sigma^{b}} \sigma^{d}=\left\{g^{a} \sigma^{b} g^{-a} \sigma^{d} \mid g \in G\right\}$.

Remark 5.7. It is tempting to extend the results for words with constants mentioned in this and in the previous sections to Lie polynomials with constants on simple Lie algebras, or, even further, to associative non-commutative polynomials on matrix algebras, in the spirit of numerous analogies described, e.g., in KBKP for genuine words (without constants). Note that the latter case naturally includes some innocent looking problems which are wide open; see, e.g., [Sl] for the case of matrix equations of the form

$$
A_{m} X^{m}+\cdots+A_{1} X+A_{0}=0 .
$$

Even the case $m=2$ of quadratic matrix equations is tricky enough, see Ge. Even stating reasonable conjectures looks as a challenge.

Acknowledgements. We thank the referees for careful reading and thoughtful remarks, which were very helpful for improving the original version.

\section{REFERENCES}

[AGKS] N. Avni, T. Gelander, M. Kassabov, A. Shalev, Word values in p-adic and adelic groups, Bull. London Math. Soc. 45 (2013), 1323-1330.

[BGG] T. Bandman, S. Garion, F. Grunewald, On the surjectivity of Engel words on PSL(2,q), Groups Geom. Dyn. 6 (2012), 409-439.

[BGK] T. Bandman, S. Garion, B. Kunyavskii, Equations in simple matrix groups: algebra, geometry, arithmetic, dynamics, Cent. Eur. J. Math. 12 (2014), 175-211.

[BGKP] T. Bandman, N. Gordeev, B. Kunyavskii, E. Plotkin, Equations in simple Lie algebras, J. Algebra 355 (2012), $67-79$.

[BK] T. Bandman, B. Kunyavskii, Criteria for equidistribution of solutions of word equations on SL(2), J. Algebra 382 (2013), 282-302.

[BZ] T. Bandman, Yu. Zarhin, Surjectivity of certain word maps on PSL(2, C) and $S L(2$, C), Eur. J. Math. 2 (2016), 614-643.

[BM] S. Bhaumik, A. Mandal, On the density of images of the power maps in Lie groups, Arch. Math. (Basel) 110 (2018), 115-130.

[Bl] H. J. Blau, A fixed-point theorem for central elements in quasisimple groups, Proc. Amer. Math. Soc. 112 (1994), 79-84.

[Bo1] A. Borel, Linear Algebraic Groups, Graduate Texts in Math., 2nd ed., Springer-Verlag, 1991.

[Bo2] A. Borel, On free subgroups of semi-simple groups, Enseign. Math. 29 (1983), 151-164.

[Bou] N. Bourbaki, Éléments de mathématiques. Groupes et algèbres de Lie, Chap. IV, V, VI, 2ème éd., Masson, Paris, 1981.

[Ch1] P. Chatterjee, On surjectivity of the power maps of solvable Lie groups, J. Algebra 248 (2002), 669-687. 
[Ch2] P. Chatterjee, On the surjectivity of the power maps of algebraic groups in characteristic zero, Math. Res. Lett. 9 (2002), 741-756.

[Ch3] P. Chatterjee, On the surjectivity of the power maps of semisimple algebraic groups, Math. Res. Lett. 10 (2003), 625-633.

[Co] B. Conrad, Reductive group schemes, in: "Autour des schémas en groupes", vol. I, Panor. Synthèses 42/43, Soc. Math. France, Paris, 2014, pp. 93-444.

[DM] S. G. Dani, A. Mandal, On the surjectivity of the power maps of a class of solvable groups, J. Group Theory 20 (2017), 1089-1101.

[ET] A. Elkasapy, A. Thom, About Gotô's method showing surjectivity of word maps, Indiana Univ. Math. J. 63 (2014), $1553-1565$.

[EG1] E. W. Ellers, N. Gordeev, On the conjectures of J. Thompson and O. Ore, Trans. Amer. Math. Soc. 350 (1998), $3657-3671$.

[EG2] E. W. Ellers, N. Gordeev, Intersection of conjugacy classes with Bruhat cells in Chevalley groups, Pacific J. Math. 214 (2004), 245-261.

[Ge] S. Gelfand, On the number of solutions of a quadratic equation, in: "Globus: General Math. Seminar", M. A. Tsfasman, V. V. Prasolov (Eds.), no. 1, Independent Univ. of Moscow, Moscow, 2004, pp. 124-133. (Russian.)

[GM] I. Z. Golubchik, A. V. Mikhalev, Generalized group identities in classical groups, Zapiski Nauchn. Sem. LOMI 114 (1982), 96-119; English transl. in J. Math. Sci. (N. Y.) 27 (1984), 2902-2918.

[Gor1] N. Gordeev, Product of conjugacy classes in algebraic groups, I, II, J. Algebra 173 (1995), 715-744, 745-779.

[Gor2] N. Gordeev, Freedom in conjugacy classes of simple algebraic groups and identities with constants, Algebra $\mathrm{i}$ Analiz 9 (1997), no. 4, 63-78; English transl. in St. Petersburg Math. J. 9 (1998), 709-723.

[Gor3] N. Gordeev, Products of conjugacy classes in perfect linear groups. Extended covering number, Zapiski Nauchn. Sem. POMI 321 (2005), 67-89; English transl. in J. Math. Sci. (N.Y.) 136 (2006), 3867-3879.

[Gor4] N. Gordeev, On Engel words on simple algebraic groups, J. Algebra 425 (2015), 215-244.

[GKP1] N. L. Gordeev, B. E. Kunyavskii, E. B. Plotkin, Word maps and word maps with constants of simple algebraic groups, Doklady Akad. Nauk 471 (2016), no. 2, 136-138; English transl. in Doklady Math. 94 (2016), 632-634.

[GKP2] N. Gordeev, B. Kunyavskii, E. Plotkin, Word maps, word maps with constants and representation varieties of one-relator groups, J. Algebra 500 (2018), 390-424.

[GS] N. Gordeev, J. Saxl, Products of conjugacy classes in Chevalley groups over local rings, Algebra i Analiz 17 (2005), no. 2, 96-107; English transl. in St. Petersburg Math. J. 17 (2006), 285-293.

[Got] M. Gotô, A theorem on compact semi-simple groups, J. Math. Soc. Japan 1 (1949), 270-272.

[GG] M. Goto, F. D. Grosshans, Semisimple Lie Algebras, Lecture Notes Pure Appl. Math., vol. 38, Marcel Dekker, INC., New York-Basel, 1978.

[Ho] G. P. Hochschild, Basic Theory of Algebraic Groups and Lie Algebras, Graduate Texts in Math., vol. 75, SpringerVerlag, Berlin-Heidelberg-New York, 1981.

[HP] D. F. Holt, W. Plesken (with an appendix by W. Hanrath), Perfect Groups, Oxford Math. Monographs, Oxford Science Publications, The Clarendon Press, Oxford Univ. Press, New York, 1989.

[HLS] C. Y. Hui, M. Larsen, A. Shalev, The Waring problem for Lie groups and Chevalley groups, Israel J. Math. 210 (2015), 81-100.

[Is] I. M. Isaacs, Commutators and the commutator subgroup, Amer. Math. Monthly 84 (1977), 720-722.

[KBKP] A. Kanel-Belov, B. Kunyavskii, E. Plotkin, Word equations in simple groups and polynomial equations in simple algebras, Vestnik St. Petersburg Univ. Math. 46 (2013), no. 1, 3-13.

[KT] A. Klyachko, A. Thom, New topological methods to solve equations over groups, Algebr. Geom. Topol. 17 (2017), 331-353.

$[\mathrm{Ku}] \quad$ B. Kunyavskii, Equations in matrix groups and algebras over number fields and rings: prolegomena to a lowbrow noncommutative Diophantine geometry, in: "Arithmetic and Geometry", L. V. Dieulefait et al. (Eds.), LMS Lecture Notes, Cambridge Univ. Press, 2015, pp. 264-282.

[LaS $\quad$ M. Larsen, A. Shalev, Fibers of word maps and some applications, J. Algebra 354 (2012), 36-48.

[LST] M. Larsen, A. Shalev, P. H. Tiep, Waring problem for finite quasisimple groups, Intern. Math. Res. Not. 2013, no. $10,2323-2348$.

[Li] M. W. Liebeck, Width questions for finite simple groups, in: "Groups St. Andrews 2013", C. M. Campbell, M. R. Quick, E. F. Robertson, C. M. Roney-Dougal (Eds.), London Math. Soc. Lecture Note Ser., vol. 422, Cambridge Univ. Press, Cambridge, 2015, pp. 51-72.

[LOST1] M. W. Liebeck, E. A. O'Brien, A. Shalev, P. H. Tiep, The Ore conjecture, J. Eur. Math. Soc. 12 (2010), 939-1008.

[LOST2] M. W. Liebeck, E. A. O'Brien, A. Shalev, P. H. Tiep, Commutators in finite quasisimple groups, Bull. Lond. Math. Soc. 43 (2011), 1079-1092.

[LM] A. Lubotzky, A. R. Magid, Varieties of Representations of Finitely Generated Groups, Mem. Amer. Math. Soc. 58 (1985), no. 336.

[Ma] G. Malle, The proof of Ore's conjecture [after Ellers-Gordeev and Liebeck-O'Brien-Shalev-Tiep], Astérisque $\mathbf{3 6 1}$ (2014), exp. no. 1069, 325-348.

[McN1] G. J. McNinch, Levi decompositions of a linear algebraic group, Transform. Groups 15 (2010), 937-964.

[McN2] G. J. McNinch, Linearity for actions on vector groups, J. Algebra 397 (2014), 666-688. 
[Mo] G. D. Mostow, Fully reducible subgroups of algebraic groups, Amer. J. Math. 78 (1956), 200-221.

[Ni] N. Nikolov, On the commutator width of perfect groups, Bull. London Math. Soc. 36 (2004), 30-36.

[Or] O. Ore, Some remarks on commutators, Proc. Amer. Math. Soc. 272 (1951), 307-314.

[PW] S. Pasiencier, H.-C. Wang, Commutators in a semi-simple Lie group, Proc. Amer. Math. Soc. 13 (1962), $907-913$.

[Re] R. Ree, Commutators in semi-simple algebraic groups, Proc. Amer. Math. Soc. 15 (1964), 457-460.

[Se] D. Segal, Words: Notes on Verbal Width in Groups, London Math. Soc. Lecture Note Ser., vol. 361, Cambridge Univ. Press, Cambridge, 2009.

[Sh1] A. Shalev, Word maps, conjugacy classes, and a non-commutative Waring-type theorem, Ann. Math. 170 (2009), 1383-1416.

[Sh2] A. Shalev, Some results and problems in the theory of word maps, in: "Erdös Centennial", L. Lovász, I. Ruzsa, V. T. Sós (Eds.), Bolyai Soc. Math. Studies, vol. 25, Springer, 2013, pp. 611-649.

[Sl] M. Slusky, Zeros of $2 \times 2$ matrix polynomials, Comm. Algebra 38 (2010), 4212-4223.

[SS] T. A. Springer, R. Steinberg, Conjugacy classes, in: "Seminar on Algebraic Groups and Related Finite Groups", Lecture Notes Math., vol. 131, Springer-Verlag, Berlin-Heidelberg-New York, 1970, pp. 167-266.

[Stei] R. Steinberg, On power maps in algebraic groups, Math. Res. Lett. 10 (2003), 621-624.

[Step1] A. Stepanov, Free product subgroups between Chevalley groups $G(\Phi, F)$ and $G(\Phi, F[t])$, J. Algebra 324 (2010), 1549-1557.

[Step2] A. Stepanov, Subring subgroups in Chevalley groups with doubly laced root systems, J. Algebra 362 (2012), 12-29.

[Th] A. Thom, Convergent sequences in discrete groups, Canad. Math. Bull. 56 (2013), 424-433.

[To] G. M. Tomanov, Generalized group identities in linear groups, Mat. Sbornik 123 (1984), no. 1, 36-49; English transl. in Math. USSR Sb. 51 (1985), 33-46.

[VO] E. B. Vinberg, A. L. Onishchik, Seminar on Lie groups and Algebraic Groups, 2nd ed., Moscow, 1995; English transl. of the 1st ed. "Lie Groups and Algebraic Groups", Springer-Verlag, Berlin-Heidelberg, 1990.

Gordeev: Department of Mathematics, Herzen State Pedagogical University, 48 Moika Embankment, 191186, St.Petersburg, RUSSia and Department of Mathematics of Sankt-Petersburg State University, 198504, Universitetsky prospekt, 28, Peterhof, St. Petersburg, RUSSiA

E-mail address: nickgordeev@mail.ru

Kunyavskir: Department of Mathematics, Bar-Ilan University, 5290002 Ramat Gan, ISRAEL

E-mail address: kunyav@macs.biu.ac.il

Plotkin: Department of Mathematics, Bar-Ilan University, 5290002 Ramat Gan, ISRAEL

E-mail address: plotkin@macs.biu.ac.il 